TRANSACTIONS OF THE

AMERICAN MATHEMATICAL SOCIETY

Volume 359, Number 2, February 2007, Pages 827-857

S 0002-9947(06)04201-2

Article electronically published on September 12, 2006

\title{
REALIZABILITY OF ALGEBRAIC GALOIS EXTENSIONS BY STRICTLY COMMUTATIVE RING SPECTRA
}

\author{
ANDREW BAKER AND BIRGIT RICHTER
}

\begin{abstract}
We discuss some of the basic ideas of Galois theory for commutative $\mathbb{S}$-algebras originally formulated by John Rognes. We restrict our attention to the case of finite Galois groups and to global Galois extensions.

We describe parts of the general framework developed by Rognes. Central rôles are played by the notion of strong duality and a trace mapping constructed by Greenlees and May in the context of generalized Tate cohomology. We give some examples where algebraic data on coefficient rings ensures strong topological consequences. We consider the issue of passage from algebraic Galois extensions to topological ones by applying obstruction theories of Robinson and Goerss-Hopkins to produce topological models for algebraic Galois extensions and the necessary morphisms of commutative $\mathbb{S}$-algebras. Examples such as the complex $K$-theory spectrum as a $K O$-algebra indicate that more exotic phenomena occur in the topological setting. We show how in certain cases topological abelian Galois extensions are classified by the same Harrison groups as algebraic ones, and this leads to computable Harrison groups for such spectra. We end by proving an analogue of Hilbert's theorem 90 for the units associated with a Galois extension.
\end{abstract}

\section{INTRODUCTION}

We discuss some ideas on Galois theory for commutative $\mathbb{S}$-algebras, also known as brave new (commutative) rings, originally formulated by John Rognes. We restrict ourselves to the case of finite Galois groups, although there are versions for profinite groups, group-like monoids, and stably dualizable topological groups. The Galois extensions which we consider are global in Rognes' terminology, i.e., we work in the stable homotopy category and not in dramatically localized versions of it.

We begin in Part 1 by describing the general framework, first outlining the generalization of 'classical' Galois theory of field extensions to commutative rings and then some of the theory developed by Rognes. In our account, central rôles are played by the notion of strong duality as discussed by Dold and Puppe [9] and a certain trace mapping constructed by Greenlees and May in the context of generalized Tate cohomology. Both of these are topological manifestations of

Received by the editors December 23, 2004.

2000 Mathematics Subject Classification. Primary 55P42, 55P43, 55S35; Secondary 55P91, 55P92, 13B05.

Key words and phrases. Commutative $S$-algebra, Galois extension, $\Gamma$-homology, Kummer theory, Picard groups.

We would like to thank John Rognes, John Greenlees, Peter Kropholler, Stefan Schwede and the referee for helpful comments. We thank the Mathematics Departments of the Universities of Glasgow and Oslo for providing us with stimulating environments to pursue this work.

(C)2006 American Mathematical Society 827

Reverts to public domain 28 years from publication 
properties of algebraic Galois extensions. We stress that this material is known to Rognes and is systematically described in [26], and the presentation reflects our approach to understanding his results rather than being original.

In the heart of our paper, Part 2, we consider the issue (raised by Rognes) of passage from algebraic Galois extensions to topological ones, i.e., given a commutative S-algebra $A$ and a $G$-Galois extension $B_{*}$ of $A_{*}=\pi_{*}(A)$ we investigate whether this extension can be realized by a Galois extension $B / A$ of $\mathbb{S}$-algebras. We focus on specific situations where we can apply the recently developed obstruction theories of Robinson and Goerss-Hopkins to produce topological models for algebraic Galois extensions and the necessary morphisms of commutative $\mathbb{S}$-algebras. In these situations, certain cohomological obstructions vanish for purely algebraic reasons related to the Galois theory of the coefficient rings of the spectra involved. However, examples such as $K U$ as a $K O$-algebra (studied in Part 1) indicate that more exotic phenomena can occur in the topological setting.

In Section 2.4 we investigate Kummer extensions of commutative $\mathbb{S}$-algebras and provide criteria that allow us to compare them with algebraic Kummer extensions. In certain cases, for instance the $C_{2}$-extensions of $K O[1 / 2]$, topological abelian Galois extensions are classified by the same Harrison groups as algebraic ones, and this leads to computable Harrison groups for such spectra. An important technical input is provided by some results about invertible module spectra and topological Picard groups proved in [5]. We end by proving an analogue of Hilbert's theorem 90 for the units associated with a Galois extension.

\section{Part 1. Galois theory for commutative rings and ring spectra}

\subsection{Galois THEORY FOR COMMUTATIVE RINGS}

We recall results on the Galois theory of commutative rings which are mainly due to Chase, Harrison and Rosenberg [7] and also described by Greither [16. The notion of Galois extensions of commutative rings was first developed by Auslander and Goldman in [3]; for further work on this see [12. It is also possible to make sense of these ideas in the context where $R$ is a commutative graded ring and $S$ is a commutative $R$-algebra, and this will be important in the topological applications.

Let $R$ be a commutative ring and $S$ a commutative $R$-algebra. Suppose that $G \leqslant \operatorname{Aut}(S / R)$, the group of all $R$-algebra automorphisms of $S$; we will indicate the (left) action of $\gamma \in G$ on $s \in S$ by writing ${ }^{\gamma} s$. We give the product $S$-algebra

$$
\prod_{\gamma \in G} S=\left\{\left(s_{\gamma}\right)_{\gamma \in G}: s_{\gamma} \in S\right\}
$$

the left $G$-action for which

$$
\alpha \cdot\left(s_{\gamma}\right)_{\gamma \in G}=\left(s_{\gamma \alpha}\right)_{\gamma \in G} \quad(\alpha \in G) .
$$

We also have the $S$-algebra of functions $f: G \longrightarrow S$ which has the left $G$-action

$$
(\alpha \cdot f)(\gamma)=f\left(\alpha^{-1} \gamma\right) \quad(\alpha \in G)
$$

There is a $G$-equivariant isomorphism of $S$-algebras

$$
\operatorname{Map}(G, S) \cong \prod_{\gamma \in G} S ; \quad f \longleftrightarrow\left(f\left(\gamma^{-1}\right)\right)_{\gamma \in G}
$$


and a unique $S$-algebra homomorphism

$$
\Theta: S \otimes_{R} S \longrightarrow \prod_{\gamma \in G} S ; \quad \Theta(u \otimes v)=\left(u^{\gamma} v\right)_{\gamma \in G} \quad(u, v \in S),
$$

which induces a $G$-equivariant homomorphism of $S$-algebras

$$
\Theta^{\prime}: S \otimes_{R} S \longrightarrow \operatorname{Map}(G, S)
$$

that will be used without further remark.

There is an isomorphism of $S[G]$-modules

$$
\Upsilon: S[G] \longrightarrow \operatorname{Map}(G, S),
$$

sending a generator $\gamma$ to the Kronecker function $\delta_{\gamma}$. Here, we use the left $S$-linear $G$-action on the group ring $S[G]$.

We denote the twisted group ring of $S$ with $G$ by $S \sharp G$; this is the free $S$-module on $G$ with product given by

$$
(s \alpha)(t \beta)=\left(s^{\alpha} t\right)(\alpha \beta) \quad(s, t \in S, \alpha, \beta \in G) .
$$

Then $S \sharp G$ is an $R$-algebra and there is an $R$-algebra homomorphism $j: S \sharp G \longrightarrow$ $\operatorname{End}_{R}(S)$ induced from the actions of $S$ and $G$ on $S$ by $R$-module homomorphisms.

Definition 1.1.1. $S / R$ is a $G$-Galois extension if it satisfies the following conditions:

(G-1) $S^{G}=R$

(G-2) $\Theta: S \otimes_{R} S \longrightarrow \prod_{\gamma \in G} S$ is an isomorphism.

Remark 1.1.2. (a) Condition (G-2) ensures that $S$ is unramified with respect to $R$. For instance, if $C_{2}$ is a cyclic group of order 2 , then $\mathbb{Z} \longrightarrow \mathbb{Z}[i]$ is not a $C_{2}$-Galois extension: because of ramification at the prime 2, the map $\Theta$ is not surjective. After inverting 2 we find that $\mathbb{Z}[1 / 2] \longrightarrow \mathbb{Z}[1 / 2, i]$ is a $C_{2}$-Galois extension. See Example 2.2.8 for related phenomena.

(b) (G-2) can also be replaced by the requirement that $\Theta^{\prime}: S \otimes_{R} S \longrightarrow \operatorname{Map}(G, S)$ is an isomorphism.

Remark 1.1.3. Later we will consider $G$-Galois extensions of graded commutative rings. By these we mean extensions of graded rings $R_{*} \longrightarrow S_{*}$ together with an action of $G \leqslant \operatorname{Aut}_{R_{*}}\left(S_{*}\right)$ such that the conditions of Definition 1.1.1 are satisfied. Note that in these cases the $G$-action preserves the grading.

Theorem 1.1.4. Let $R$ be a commutative ring and let $S$ be a commutative $R$ algebra with $G \leqslant \operatorname{Aut}_{R}(S)$ and assume that $S^{G}=R$. Then the following conditions are equivalent:

(a) $S / R$ is a $G$-Galois extension.

(b) $\Theta: S \otimes_{R} S \longrightarrow \prod_{\gamma \in G} S$ is an epimorphism.

(c) There are finite sequences $u_{1}, \ldots, u_{n}, v_{1}, \ldots, v_{n} \in S$ for which

$$
\sum_{i=1}^{n} u_{i}{ }^{\gamma} v_{i}= \begin{cases}1 & \text { if } \gamma=1, \\ 0 & \text { otherwise } .\end{cases}
$$

(d) $S$ is a finitely generated projective $R$-module and $j: S \sharp G \longrightarrow \operatorname{End}_{R}(S)$ is an isomorphism. 
We define the trace of $S / R$ to be the $R$-module homomorphism

$$
\operatorname{tr}_{S / R}: S \longrightarrow R ; \quad \operatorname{tr}_{S / R}(s)=\sum_{\gamma \in G} \gamma_{s}
$$

We will write $\operatorname{tr}=\operatorname{tr}_{S / R}$ when no ambiguity is likely to result.

Recall that a ring is connected if its only idempotents are 0 and 1 . Also recall that given a left $R[G]$-module $M, \operatorname{Hom}_{R}(M, R)$ can be viewed as a left $R[G]$-module with the contragredient $G$-action determined by

$$
(\gamma \cdot f)(m)=f\left(\gamma^{-1} m\right) \quad\left(f \in \operatorname{Hom}_{R}(M, R), \gamma \in G, m \in M\right) .
$$

Theorem 1.1.5. For a $G$-Galois extension $S / R$, the following hold:

(a) $S$ is faithfully flat over $R$.

(b) $\operatorname{tr}: S \longrightarrow R$ is an epimorphism.

(c) The unit $R \longrightarrow S$ is a split monomorphism of $R$-modules.

(d) If $R$ and $S$ are both connected, then $\operatorname{Aut}(S / R)=G$.

(e) For any commutative $R$-algebra $T, T \otimes_{R} S / T$ is a $G$-Galois extension.

(f) $S$ is a finitely generated projective invertible $R[G]$-module, hence it is of constant rank 1. Furthermore, $S$ is self-dual, i.e., $S^{*}=\operatorname{Hom}_{R}(S, R) \cong S$ as $R[G]$-modules.

Remark 1.1.6. (i) The proof of (f) makes use of the trace pairing

$$
S \otimes_{R} S \stackrel{\text { mult }}{\longrightarrow} S \stackrel{\operatorname{tr}_{S / R}}{\longrightarrow} R
$$

to establish the self-duality and projectivity (see [7, Theorem 4.2]). In Section 1.3 we will discuss a more abstract setting in which such duality occurs; in particular, the self-duality of a Galois extension forces it to be self-dual in the categorical sense we will describe (this is a special case of [22, Lemma 2.9], where the relationship between these notations is also studied in detail).

(ii) When $R$ contains non-trivial idempotents, Theorem 1.1.5(d) need not be true. For example, if $G$ has order 2 and $S=R \times R$ is the trivial $G$-extension, then given any non-trivial idempotent $e \in R$, the map

$$
\varphi: R \times R \longrightarrow R \times R ; \quad \varphi(x, y)=(x e+y(1-e), x(1-e)+y e)
$$

is an $R$-algebra isomorphism which is not induced by an element of $G$.

Condition (f) has an important group cohomological consequence. First recall the following well-known observation about group cohomology (for example, see [28, Example 6.1.2]).

Lemma 1.1.7. For any ring $R$ and $R[G]$-module $M$,

$$
\mathrm{H}^{*}(G ; M)=\operatorname{Ext}_{\mathbb{Z} G}^{*}(\mathbb{Z}, M) \cong \operatorname{Ext}_{R[G]}^{*}(R, M) .
$$

Then we have

Proposition 1.1.8. Let $S / R$ be a $G$-Galois extension. Then

$$
\mathrm{H}^{*}(G ; S)=\mathrm{H}^{0}(G ; S)=R \text {. }
$$

Proof. By Lemma 1.1.7,

$$
\mathrm{H}^{*}(G ; S)=\operatorname{Ext}_{R[G]}^{*}(R, S) .
$$

Recall from Theorem 1.1.5(f) that $S$ is finitely generated, self-dual and projective as an $R[G]$-module, and it is also finitely generated and projective as an $R$-module. As 
$S$ is a retract of a finitely generated free $R[G]$-module, it suffices to prove the claim for $R[G]$. As $R[G] \cong \operatorname{Map}(G, R)$, an adjunction argument proves the claim.

For further results, as for instance the fundamental theorem of Galois theory in this context see [7, 16, 12. We follow [16] in making Definitions 1.1.9] and 1.1.11 below.

Definition 1.1.9. For a commutative ring $R$ and a finite group $G$, let $\operatorname{Gal}(R, G)$ denote the category of $G$-Galois extensions of $R$ with morphisms the $R$-algebra homomorphisms commuting with the actions of $G$.

Proposition 1.1.10. If $S / R$ and $T / R$ are two $G$-Galois extensions and $\varphi: S \longrightarrow T$ is an R-algebra homomorphism commuting with the actions of $G$, then $\varphi$ is an isomorphism. Hence $\operatorname{Gal}(R, G)$ is a (large) groupoid.

Proof. The proof of [16, Proposition 0.1.12] only applies when $R$ has no non-trivial idempotents, so for completeness we prove the general case.

First note that by parts (a) and (e) of Theorem 1.1.5, it suffices to replace $S / R$ and $T / R$ by $\left(S \otimes_{R} T \otimes_{R} S\right) /\left(S \otimes_{R} T\right)$ and $\left(S \otimes_{R} T \otimes_{R} T\right) /\left(S \otimes_{R} T\right)$, and then note that

$$
S \otimes_{R} T \otimes_{R} S \cong \prod_{\gamma \in G} S \otimes_{R} T \cong S \otimes_{R} T \otimes_{R} T .
$$

Thus we might as well assume that $S=T=\prod_{G} R$ is the trivial $G$-Galois extension. For each $\alpha \in G$, there is an idempotent $e_{\alpha}=\left(\delta_{\alpha \gamma}\right) \in S$.

Now let

$$
e_{1}^{\prime}=\varphi\left(e_{1}\right)=\sum_{\gamma \in G} t_{\gamma} e_{\gamma}
$$

with $t_{\gamma} \in R$. Then as $\varphi$ commutes with the action of elements of $G$, for each $\alpha \in G$ we have

$$
e_{\alpha}^{\prime}=\varphi\left(e_{\alpha}\right)=\varphi\left(\alpha \cdot e_{1}\right)=\alpha \varphi\left(e_{1}\right)=\sum_{\gamma \in G} t_{\alpha^{-1} \gamma} e_{\gamma} .
$$

Thus the $e_{\alpha}^{\prime}$ form a complete set of orthogonal idempotents in $S$. Note that the $e_{\alpha}$ also form a basis for the free $R$-module $S$. The equation $e_{1}^{\prime} e_{1}^{\prime}=e_{1}^{\prime}$ then shows that $t_{\gamma}^{2}=t_{\gamma}$ and

$$
\sum_{\beta} t_{\beta}=\sum_{\beta} t_{\beta} \sum_{\gamma} e_{\gamma}=\sum_{\alpha, \gamma} t_{\alpha^{-1} \gamma} e_{\gamma}=\sum_{\alpha} e_{\alpha}^{\prime}=1
$$

proves that the $t_{\alpha}$ also form a complete set of orthogonal idempotents in $R$.

Now for elements $s \in S$ and $x_{\alpha} \in R$, consider the equation

$$
\sum_{\alpha \in G} x_{\alpha} e_{\alpha}^{\prime}=s
$$

which is equivalent to

$$
\sum_{\alpha \in G} \sum_{\gamma \in G} x_{\alpha} t_{\alpha^{-1} \gamma} e_{\gamma}=s .
$$

Multiplying by $e_{\beta}$ we obtain $x_{\alpha} t_{\alpha^{-1} \beta} e_{\beta}=r_{\beta} e_{\beta}$, where $r_{\beta} \in R$ is the unique element for which $s e_{\beta}=r_{\beta} e_{\beta}$. Thus for $\alpha, \beta \in G$ we have $x_{\alpha} t_{\alpha^{-1} \beta}=r_{\beta}$. Multiplying by $t_{\alpha^{-1} \beta}$ now gives $x_{\alpha} t_{\alpha^{-1} \beta}=r_{\beta} t_{\alpha^{-1} \beta}$. Summing over $\beta \in G$ we now obtain $x_{\alpha}=\sum_{\beta \in G} r_{\beta} t_{\alpha^{-1} \beta}$, since $\sum_{\beta \in G} t_{\alpha^{-1} \beta}=\sum_{\gamma \in G} t_{\gamma}=1$. Thus (1.1.4) has the unique solution given by this formula. Hence $\varphi$ is an isomorphism. 
Because of the last result, we may define an equivalence relation $\sim$ on the objects of $\operatorname{Gal}(R, G)$ by requiring that $S / R \sim T / R$ if and only if there is a morphism $\varphi: S \longrightarrow T$ in $\operatorname{Gal}(R, G)$. The equivalence classes are then the isomorphism classes of $G$-Galois extensions of $R$.

Definition 1.1.11 (See [17] and [16, 3.2]). The Harrison set $\operatorname{Har}(R, G)$ is the set of isomorphism classes of $G$-Galois extensions of $R$. When $G$ is abelian, this is naturally an abelian group often called the Harrison group.

There are some useful properties of this construction that will be required later. Details can be found in [17, Theorem 4] or [16, Theorems 3.2, 3.3 and 3.5] or supplied by the reader.

Proposition 1.1.12. Let $R$ be a commutative ring.

(a) $\operatorname{Har}(R,-)$ defines a covariant functor

$$
\operatorname{Har}(R,-): \text { FinAbGps } \rightsquigarrow \text { AbGps }
$$

from finite abelian groups to abelian groups.

(b) $\operatorname{Har}(R,-)$ is left exact and is pro-representable.

(c) $\operatorname{Har}(R,-)$ preserves products, i.e., for finite abelian groups $G$ and $H$ there is a natural isomorphism

$$
\operatorname{Har}(R, G \times H) \cong \operatorname{Har}(R, G) \times \operatorname{Har}(R, H) .
$$

Of course, part (c) implies that $\operatorname{Har}(R,-)$ is determined by its values on cyclic groups.

\subsection{Abelian extensions and Kummer theory}

In [17, 15, 16, a theory of abelian extensions of commutative rings was described, including an analogue of Kummer theory. We will describe this algebraic theory, and in Section 2.4 a topological analogue will be introduced. Our goal is to show how $\operatorname{Har}(R, G)$ can be determined under certain conditions, making use of Proposition 1.1.12, which reduces the problem to the case of cyclic groups.

Let $R$ be a commutative ring containing $1 / n$ and a primitive $n$-th root of unity $\zeta$. For a unit $u \in R^{\times}$, we set

$$
R(n ; u)=R[x] /\left(x^{n}-u\right),
$$

where $x$ is an indeterminate. We will write $\bar{z}$ for the coset

$$
z+\left(x^{n}-u\right) \in R[x] /\left(x^{n}-u\right) .
$$

Of course, for any $t \in R^{\times}$there is a canonical $R$-algebra isomorphism

$$
R\left(n ; t^{n} u\right) \stackrel{\cong}{\rightrightarrows} R(n ; u) ; \quad \overline{t x} \mapsto \bar{x} .
$$

Note that $R(n ; u) / R$ is a $C_{n}$-Galois extension, where the action of the generator $\gamma_{n} \in C_{n}$ is given by

$$
\gamma_{n} \cdot \bar{x}=\overline{\zeta x}
$$

The set $\operatorname{Kum}_{n}(R)$ of $R$-algebra isomorphism classes of such $R(n ; u)$ is an abelian group with product on isomorphism classes given by

$$
[R(n ; u)][R(n ; v)]=[R(n ; u v)]
$$


and whose unit is the class $[R(n ; 1)]$, where

$$
R(n ; 1)=R[x] /\left(x^{n}-1\right)=\prod_{i=1}^{n} R[x] /\left(x-\zeta^{i}\right)=\prod_{\gamma \in G} R
$$

is the trivial $G$-Galois extension. Of course, $\operatorname{Kum}_{n}(R) \leqslant \operatorname{Har}\left(R, C_{n}\right)$. In fact there is an isomorphism of groups

$$
R^{\times} /\left(R^{\times}\right)^{n} \cong \operatorname{Kum}_{n}(R) ; \quad u\left(R^{\times}\right)^{n} \longmapsto[R(n ; u)] .
$$

Now let $S / R$ be a $C_{n}$-Galois extension. For $k=0,1, \ldots, n-1$, let

$$
S^{(k)}=\left\{s \in S:{ }^{\gamma_{n}} s=\zeta^{k} s\right\} \subseteq S .
$$

Then each $S^{(k)}$ is an $R$-submodule of $S$ and is a summand, hence it is finitely generated projective. Furthermore, the product in $S$ gives rise to isomorphisms $S^{(k)} \otimes_{R} S^{(\ell)} \longrightarrow S^{(k+\ell)}$. In particular we obtain

$$
\overbrace{S^{(1)} \otimes_{R} \cdots \otimes_{R} S^{(1)}}^{n} \cong S^{(1)} \otimes_{R} S^{(n-1)} \stackrel{\cong}{\rightrightarrows} S^{(0)}=R .
$$

This shows that $S^{(1)}$ is an invertible $R$-module which represents an element $\left[S^{(1)}\right]$ of the Picard group $\operatorname{Pic}(R)$ whose order is a divisor of $n$; we write

$$
\operatorname{Pic}(R)[n]=\left\{P \in \operatorname{Pic}(R): P^{n}=1\right\} .
$$

Thus there is a group homomorphism

$$
\operatorname{Har}\left(R, C_{n}\right) \longrightarrow \operatorname{Pic}(R)[n] ; \quad[S] \longmapsto\left[S^{(1)}\right] .
$$

Now from [15, 16] we have

Proposition 1.2.1. There is an exact sequence of abelian groups

$$
1 \rightarrow R^{\times} /\left(R^{\times}\right)^{n} \longrightarrow \operatorname{Har}\left(R, C_{n}\right) \longrightarrow \operatorname{Pic}(R)[n] \rightarrow 1 .
$$

There is a generalization of these ideas to the case where $G$ is any finite abelian group and $R$ contains $1 /|G|$ as well as a primitive $d$-th root of unity $\zeta$, where $d=\operatorname{lcm}\{|\gamma|: \gamma \in G\}$ is the exponent of $G$. Presumably the following is known to experts (it is hinted at in [15, 16]), but we give details since we know of no convenient reference.

First note that as an $R[G]$-module, the group $\operatorname{ring} R[G]$ has a decomposition

$$
R[G]=\bigoplus_{\chi} R[G] e_{\chi}
$$

where the sum is over the characters $\chi \in \operatorname{Hom}(G,\langle\zeta\rangle)=\operatorname{Hom}\left(G, R^{\times}\right)$. This decomposition is effected by the orthogonal idempotents

$$
e_{\chi}=\frac{1}{|G|} \sum_{\gamma \in G} \chi\left(\gamma^{-1}\right) \gamma \in R[G]
$$

which decompose 1 . It is easily seen by direct calculation that the $R$-module $R[G] e_{\chi}=e_{\chi} R[G]$ is free of rank 1 .

Now for a $G$-Galois extension $S / R$ as above there is a decomposition of $R[G]$ modules

$$
S=\bigoplus_{\chi} S(\chi)
$$

where $S(\chi)=e_{\chi} S$. 
Lemma 1.2.2. For characters $\chi_{1}, \chi_{2}$, the multiplication map $S\left(\chi_{1}\right) \otimes_{R} S\left(\chi_{2}\right) \rightarrow$ $S\left(\chi_{1} \chi_{2}\right)$ is an isomorphism. Hence for each character $\chi, S(\chi)$ is an invertible $R$-module.

Proof. This is similar to the proof for the case of a cyclic group. The invertibility comes about because each character $\chi$ has an inverse character $\bar{\chi}$ defined by $\bar{\chi}(\gamma)=$ $\chi(\gamma)^{-1}$.

The character group of $G$ is the abelian group $G^{\circ}=\operatorname{Hom}(G, \mathbb{Q} / \mathbb{Z})$. Then $G^{\circ}$ is finite of order $|G|$ and $G^{\circ} \cong \operatorname{Hom}(G,\langle\zeta\rangle)$. Now set

$$
\operatorname{Pic}(R, G)=\operatorname{Hom}\left(G^{\circ}, \operatorname{Pic}(R)\right)=\operatorname{Hom}\left(G^{\circ}, \operatorname{Pic}(R)[d]\right) .
$$

In order to give an estimate of $\operatorname{Har}(R, G)$, we state the following result. As we will not use this result later on, we refrain from giving a proof.

Proposition 1.2.3. When $G$ is abelian, there is a natural exact sequence of abelian groups

$$
0 \rightarrow \mathrm{H}^{2}\left(G^{\circ}, R^{\times}\right) \longrightarrow \operatorname{Har}(R, G) \longrightarrow \operatorname{Pic}(R, G) \rightarrow 0 .
$$

We now briefly discuss the graded version of Kummer theory. For a graded commutative ring $R_{*}$, we have to distinguish between the cases where the characteristic is two and the general case. In the former case the grading is easily dealt with, so we concentrate on cases where two is not zero. Then the units of $R_{*}$ are in even degrees only.

If we want to build the analogue of $R(n ; u)$ for a graded ring $R_{*}$, we need to assume that the degree of $u \in R_{*}^{\times}$is divisible by $2 n$. Let $R_{r *}$ be the subring of $R_{*}$ of elements in degrees divisible by $r$. We can still identify $\operatorname{Kum}_{n}\left(R_{*}\right)$ with a quotient of units

$$
R_{2 n *}^{\times} /\left(R_{2 *}^{\times}\right)^{n} \cong \operatorname{Kum}_{n}\left(R_{*}\right) .
$$

We still obtain an eigenspace decomposition of every element in $\operatorname{Har}\left(R_{*}, C_{n}\right)$ and therefore every $C_{n}$-Galois extension gives rise to a graded invertible module over $R_{*}$ of order dividing $n$. Therefore we get a left-exact sequence

$$
1 \rightarrow R_{2 n *}^{\times} /\left(R_{2 *}^{\times}\right)^{n} \longrightarrow \operatorname{Har}\left(R_{*}, C_{n}\right) \longrightarrow \operatorname{Pic}\left(R_{*}\right)[n],
$$

but not every element in $\operatorname{Pic}\left(R_{*}\right)[n]$ has to be in the image of $\operatorname{Har}\left(R_{*}, C_{n}\right)$. For instance if $R_{*}$ is periodic such that $R_{*+n}=R_{*}$ with $n$ even, then $\Sigma R_{*}$ is in $\operatorname{Pic}\left(R_{*}\right)[n]$ but cannot come from a $C_{n}$-extension. However, if we restrict our attention to elements in the Picard group which are concentrated in even degrees and of order dividing $n$, the construction given in [16, p. 22] ensures that such elements stem from the Harrison group.

\subsection{Duality in a Symmetric monoidal Category}

The Galois theory for commutative rings of Section 1.1 has some crucial aspects which can be generalized to the context of symmetric monoidal categories. At the heart of this are the notions of strong duality and self-duality, both of which are visible in the above account. The appropriate notions are described in detail in [9], and some aspects appear in [18, 19. We give an account based on [9] but with some modifications of notation.

Let $\mathscr{C}$ be a closed symmetric monoidal category with multiplication $\otimes$, twist map $\tau$ and unit $I$. We denote the internal homomorphism object on $X, Y \in \mathscr{C}$ by 
$F(X, Y)$ and write $D X$ for $F(X, I)$. For every $X \in \mathscr{C}$ we have a canonical evaluation morphism $\varepsilon=\varepsilon_{X}: D X \otimes X \longrightarrow I$ which corresponds to id $\operatorname{id}_{X} \in \mathscr{C}(X, X) \cong$ $\mathscr{C}(D X \otimes X, I)$. An object $X$ is weakly self-dual if $X$ is isomorphic to $D X$. There is always a map $\delta=\delta_{X}: X \longrightarrow D D X$ which corresponds to

$$
X \otimes D X \underset{\cong}{\stackrel{\tau}{\cong}} D X \otimes X \stackrel{\varepsilon}{\rightarrow} I .
$$

Definition 1.3.1. An object $X$ is reflexive if $\delta_{X}$ is an isomorphism.

We may define $\mu=\mu_{X Y}: D X \otimes D Y \longrightarrow D(Y \otimes X)$ corresponding to the composite

$$
D X \otimes D Y \otimes Y \otimes X \stackrel{\mathrm{id} \otimes_{\varepsilon_{Y}} \otimes \mathrm{id}}{\longrightarrow} D X \otimes I \otimes X \cong D X \otimes X \stackrel{\varepsilon_{X}}{\longrightarrow} I .
$$

Definition 1.3.2. An object $X$ is strongly dualizable if it is reflexive and $\mu_{X} D X$ is an isomorphism. This condition is equivalent to the requirement that the composition

$$
D X \otimes X \stackrel{\mathrm{id} \otimes \delta_{X}}{\longrightarrow} D X \otimes D D X \stackrel{\mu}{\longrightarrow} D(D X \otimes X)
$$

be an isomorphism, and this means that $D X \otimes X$ is canonically weakly self-dual.

If $X$ is strongly dualizable and weakly self-dual, we call $X$ strongly self-dual.

When $X$ is strongly dualizable, the coevaluation $\eta=\eta_{X}: I \longrightarrow X \otimes D X$ is the composite

$$
I=D I \stackrel{D \varepsilon}{\longrightarrow} D(D X \otimes X) \stackrel{\mu^{-1}}{\cong} D X \otimes D D X \underset{\mathrm{id} \otimes \delta^{-1}}{\cong} D X \otimes X \underset{\cong}{\cong} X \otimes D X .
$$

The following result taken from [9, Theorem 1.3] summarizes the main properties of duality.

Theorem 1.3.3. Let $X$ be an object of $\mathscr{C}$ and $\varepsilon: D X \otimes X \longrightarrow I$ the evaluation. Then the following conditions are equivalent:

(a) $X$ is strongly dualizable.

(b) There is a morphism $\eta: I \longrightarrow X \otimes D X$ for which the following compositions are the identity morphisms $\operatorname{id}_{X}$ and $\operatorname{id}_{D X}$, respectively:

$$
\operatorname{id}_{X}: X \cong I \otimes X \stackrel{\eta \otimes \operatorname{id}_{X}}{\longrightarrow} X \otimes D X \otimes X \stackrel{\operatorname{id}_{X} \bowtie \varepsilon}{\longrightarrow} X \otimes I \cong X,
$$

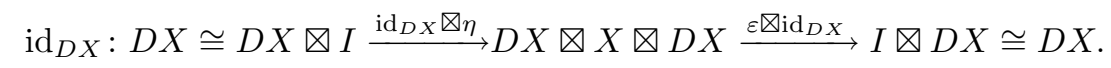

(c) For every pair of objects $U$ and $V$, the map

$$
\varphi_{U V}: \mathscr{C}(U, V \otimes D X) \rightarrow \mathscr{C}(U \otimes X, V),
$$

which sends $f: U \longrightarrow V \otimes D X$ to the composite

$$
U \otimes X \stackrel{f \bowtie \mathrm{id}_{X}}{\longrightarrow} V \otimes D X \otimes X \stackrel{\mathrm{id}_{V} \bowtie \varepsilon}{\longrightarrow} V \otimes I \cong V,
$$

is a bijection.

Furthermore, if these conditions are satisfied, then the morphism $\eta$ of (b) is necessarily the coevaluation and the bijection $\varphi_{U V}$ of (c) sends $\eta$ to the composition $I \otimes X \cong X \stackrel{\mathrm{id}_{X}}{\longrightarrow} X$.

We end this section with a useful result mentioned in the proof of 21, Theorem XVI 7.4]. 
Proposition 1.3.4. Let $\mathscr{C}$ be closed and let $X$ be strongly dualizable in $\mathscr{C}$. Suppose that $R$ is a retract of $X$ with maps $j: R \longrightarrow X$ and $r: X \longrightarrow R$ satisfying $r j=$ $\operatorname{id}_{R}: R \longrightarrow R$. Then $R$ is strongly dualizable with the natural evaluation $\varepsilon^{\prime}: D R \otimes$ $R \longrightarrow I$ and coevaluation given by the composite

$$
\eta^{\prime}: I \stackrel{\eta}{\rightarrow} X \otimes D X \stackrel{r \unrhd j^{*}}{\longrightarrow} R \otimes F(R, I),
$$

where $j^{*}: D X \longrightarrow D R$ is dual to $j: R \longrightarrow X$.

\subsection{Brave new Galois extensions}

The notion of a Galois extension in the context of the commutative $\mathbb{S}$-algebras of [11] was introduced by John Rognes. We restrict our attention to the case of finite Galois groups, and all Galois extensions which we consider are 'global', i.e., we work in the unlocalized setting.

Given a commutative $\mathbb{S}$-algebra $A$, we will work in the categories of $A$-modules $\mathscr{M}_{A}$ and its derived category $\mathscr{D}_{A}$. These categories are complete and symmetric monoidal under the smash product $\wedge_{A}$. In $\mathscr{D}_{A}$, an $A$-module $L$ has as a weak dual $D_{A} L=F_{A}(L, A)$.

Then $L$ is strongly dualizable if for every $A$-module $M, F_{A}(L, M) \sim F_{A}(L, A) \wedge_{A}$ $M$, while $L$ is strongly self-dual if in addition $F_{A}(L, A) \sim L$.

The following useful result on strongly dualizable objects in $\mathscr{D}_{A}$ is taken from [18, §2] (see also [21] and [22]).

Proposition 1.4.1. Let $X$ be an A-module. Then $X$ is strongly dualizable in $\mathscr{D}_{A}$ if and only if it is weakly equivalent to a retract of a finite cell $A$-module.

Our next definition is of course suggested by the algebraic notion of faithful flatness.

Definition 1.4.2. An $A$-module $N$ is faithful (as an $A$-module) if whenever $M$ is an $A$-module for which $N \wedge_{A} M \sim *$, then $M \sim *$.

Remark 1.4.3. If $N$ is faithful, then the homology theory $N_{*}^{A}(-)=\pi_{*}\left(N \wedge_{A}-\right)$ detects weak equivalences since a morphism of $A$-modules $f: M \longrightarrow M^{\prime}$ is a weak equivalence if and only if the induced homomorphism $f_{*}: N_{*}^{A} M \longrightarrow N_{*}^{A} M^{\prime}$ is an isomorphism.

We can now give the key definition of a Galois extension essentially due to Rognes [26].

Definition 1.4.4. Let $A$ be a commutative $\mathbb{S}$-algebra and let $B$ be a commutative cofibrant $A$-algebra. Let $G$ be a finite (discrete) group and suppose that there is an action of $G$ on $B$ by commutative $A$-algebra morphisms. Then $B / A$ is a weak $G$-Galois extension if it satisfies the following two conditions:

(BNG-1) The natural map $A \longrightarrow B^{\mathrm{h} G}=F\left(E G_{+}, B\right)^{G}$ is a weak equivalence of $A$-algebras.

(BNG-2) There is a natural equivalence of $B$-algebras $\Theta: B \wedge_{A} B \stackrel{\sim}{\longrightarrow} F\left(G_{+}, B\right)$ induced from the action of $G$ on the right hand factor of $B$.

$B / A$ is a $G$-Galois extension if it also satisfies

(BNG-3) $B$ is faithful as an $A$-module. 
In fact Rognes does not insist on (BNG-3), but calls extensions satisfying (BNG1) and (BNG-2) Galois extensions and adds faithfulness as a requirement whenever needed. So far, there are no known examples of Galois extensions which are not faithful.

In (BNG-2), we use the topological analogue of the map $\Theta$ from (1.1.2). We also consider the maps $\widetilde{\gamma}$ defined in (1.4.5). These have product

$$
B \wedge_{A} B \longrightarrow \prod_{\gamma \in G} B
$$

The following base-change results can be found in [26, $\S 7]$.

Proposition 1.4.5. Let $A$ be a commutative $\mathbb{S}$-algebra and let $A \longrightarrow B$ and $A \longrightarrow$ $C$ be maps of commutative $\mathbb{S}$-algebras.

(a) $C \wedge_{A} B$ admits a canonical commutative $C$-algebra structure.

(b) If $G$ acts on $B$ by A-algebra morphisms, then there is a canonical extension of the action of $G$ on $B$ to one by $C$-algebra morphisms on $C \wedge_{A} B$.

(c) If $G$ acts on $B$ by A-algebra morphisms and $C$ is strongly self-dual in $\mathscr{D}_{A}$ and $C$ is faithful as an $A$-module, then $B / A$ is a $G$-Galois extension if and only if $C \wedge_{A} B / C$ is a $G$-Galois extension.

Here are some examples. Proofs that these are actually Galois extensions can be found in [26].

Example 1.4.6. For a commutative $\mathbb{S}$-algebra $A$ and finite group $G$, the morphism

$$
A \longrightarrow F\left(G_{+}, A\right) \cong \prod_{\gamma \in G} A
$$

induced from the trivial action of $G$ on $A$ is the trivial $G$-Galois extension.

Example 1.4.7. Let $R \longrightarrow S$ be a $G$-Galois extension in the algebraic sense of Section 1.1. Then the natural morphism of Eilenberg-Mac Lane spectra $H R \longrightarrow$ $H S$ makes $H S / H R$ into a $G$-Galois extension.

Example 1.4.8. Let $E G$ be any contractible space on which $G$ acts freely. Let $B / A$ be a $G$-Galois extension. Then $F\left(E G_{+}, B\right) / A$ is a $G$-Galois extension, and the collapse map $E G_{+} \longrightarrow S^{0}$ induces a morphism of $A$-algebras $B \longrightarrow F\left(E G_{+}, B\right)$ commuting with the actions of $G$ and which is an equivalence of $B$-algebras.

Example 1.4.9. Let $\iota: K O \longrightarrow K U$ be the complexification morphism which can be given the structure of a morphism of commutative $\mathbb{S}$-algebras.

The action of $C_{2}=\left\langle\gamma_{2}\right\rangle$ originates in the action of the stable operation $\psi^{-1}$ whose action on $K U_{2 n}=\mathbb{Z} u^{n}$ satisfies

$$
\gamma_{2} \cdot u^{n}=\psi^{-1}\left(u^{n}\right)=(-1)^{n} u^{n} .
$$

Recall that

$$
K O_{*}=\mathbb{Z}\left[\eta, y, w, w^{-1}\right] /\left(2 \eta, \eta^{3}, y \eta, y^{2}-4 w\right),
$$

where $\eta \in K O_{1}, y \in K O_{4}$ and $w \in K O_{8}$.

By the 'Theorem of Reg Wood' [1, p. 206], multiplication by the non-zero element $\eta \in K O_{1}$ induces a cofibre sequence of $K O$-modules

$$
\Sigma K O \stackrel{\eta}{\longrightarrow} K O \longrightarrow K O \wedge \operatorname{cone}(\eta)
$$


in which $K O \wedge \operatorname{cone}(\eta) \sim K U$ as $K O$-modules. This makes it clear that $K U$ is selfdual in $\mathscr{D}_{K O}$ since in $\mathscr{D}_{\mathbb{S}}$ we have $D$ cone $(\eta) \sim \Sigma^{-2}$ cone $(\eta)$. Then as $K U$-modules, $K U \wedge_{K O} K U \sim K U \wedge \operatorname{cone}(\eta)$. Using this equivalence, Rognes shows in 26] that (BNG-2) holds.

Note that although $K U_{*}$ is not a projective module over $K O_{*}, K U$ is in fact faithful over $K O$ since if $M$ is a $K O$-module with $K U \wedge_{K O} M \sim *$, then using the cofibre sequence

$$
\Sigma K O \wedge_{K O} M \stackrel{\eta \wedge \mathrm{id}}{\longrightarrow} K O \wedge_{K O} M \longrightarrow K U \wedge_{K O} M
$$

we see that $\Sigma K O \wedge_{K O} M \stackrel{\eta \wedge \text { id }}{\longrightarrow} K O \wedge_{K O} M \cong M$ is a weak equivalence. Now since $\eta \in K O_{1}$ is nilpotent, this implies that $M \sim *$.

In the following, we state and prove some basic results about Galois extensions which we will need later. These were stated by J. Rognes around 2000, and proofs can now be found in [26, Lemmas 6.1.2, 6.4.3, Proposition 6.4.7].

Theorem 1.4.10. Let $A$ and $B$ be commutative $\mathbb{S}$-algebras and let $A \longrightarrow B$ be a $G$-Galois extension. Then in the derived category of $A$-modules $\mathscr{D}_{A}$, the following hold:

(a) $B$ is strongly self-dual.

(b) For every $B$-module $N, N \wedge_{A} B \sim F\left(G_{+}, N\right)$.

(c) For every $B$-module $N, N \wedge G_{+} \sim F_{A}(B, N)$. In particular, $B \wedge G_{+} \sim$ $F_{A}(B, B)$.

Proof. In the proof we will make extensive use of notions developed in Section 1.3. The key part is (a), the others follow by formal arguments involving strong duality, and we omit these.

The idea is to emulate as far as possible the ideas used in proving the algebraic results of Section 1.1. The most important ingredient is a (weak) trace morphism

$$
B \longrightarrow B^{\mathrm{h} G} \stackrel{\sim}{\longrightarrow} A
$$

which factorizes the symmetrization map $\sum_{\gamma \in G} \gamma: B \longrightarrow B$. A construction for such a map can be found in [14, Theorem 5.10]. In our context (with $B_{G}$ denoting the naive $G$-spectrum associated with $B$ ), this produces a homotopy commutative diagram

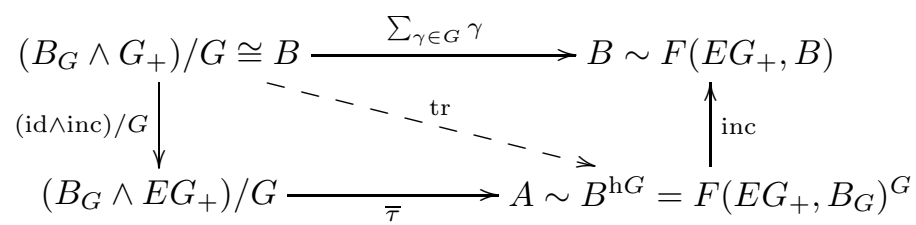

and we take for our trace map the composition

$$
\operatorname{tr}=\operatorname{tr}_{B / A}=\bar{\tau} \circ(\text { id } \wedge \text { inc }) / G: B \longrightarrow A .
$$

It is straightforward to check that this is in fact a morphism of $A$-modules. Having obtained a trace map, we can now define an evaluation map to be the trace pairing

$$
\varepsilon: B \wedge_{A} B \stackrel{\text { mult }}{\longrightarrow} B \stackrel{\text { tr }}{\longrightarrow} A
$$

which is a morphism of $A$-modules. 
Remarks 1.4.11.

(i) If $B_{G}$ is $G$-equivariantly of the form $A \wedge G_{+}$, then it is known from [14, Proposition 2.4] that the map $\bar{\tau}$ in (1.4.3) is an isomorphism.

(ii) If $N \triangleleft G$, then there is a homotopy factorization

$$
\operatorname{tr}_{B / A} \sim \operatorname{tr}_{B / B^{\mathrm{h} N}} \operatorname{tr}_{B^{\mathrm{h} N} / A} .
$$

We are grateful to J. Greenlees for showing us a verification of this formula.

(iii) We claim that the trace map $\operatorname{tr}: B \longrightarrow A$ is $G$-invariant in the sense that for any $\gamma \in G$, the composition

$$
B \stackrel{\gamma}{\rightarrow} B \stackrel{\text { tr }}{\longrightarrow} A
$$

is homotopic to tr.

Using the description in [14 pp. 38-42] we can write the trace map as the composition

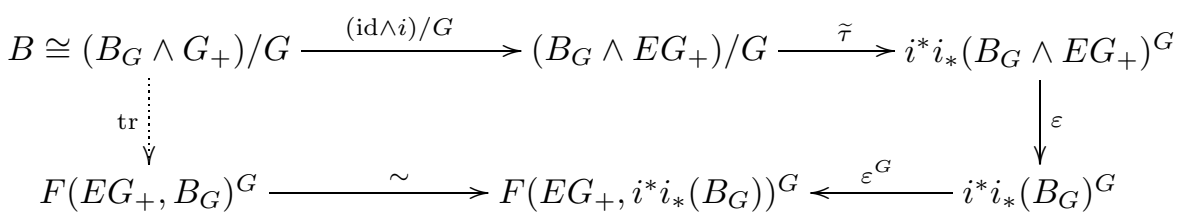

We should mention that there is a $G$-action on $\left(B_{G} \wedge E G_{+}\right) / G$. We consider the semidirect product $G \ltimes G$, where we take the conjugation action of $G$ on itself. Then $G \ltimes G$ acts on $B_{G} \wedge E G_{+}$, and if we divide out by the normal subgroup, we are still left with a $G$-action on the quotient. This is the $G$-action that Greenlees and May use on $\left(B_{G} \wedge E G_{+}\right) / G$ (see [14, p. 38]).

The map $\widetilde{\tau}$ is a transfer map and is natural, hence it is equivariant. We denote by $\varepsilon$ maps induced by the collapse map $E G_{+} \longrightarrow S^{0}$. The last map is the unit of the adjunction $\left(i^{*}, i_{*}\right)$ defined in [14, Lemma 0.1]; this is an equivariant map although it is not a weak equivalence in the equivariant setting. Therefore the trace is $G$-invariant as claimed, and hence the self-duality of Theorem 1.4 .10 (a) is given by a $G$-equivariant equivalence $B \longrightarrow F_{A}(B, A)$. This map is adjoint to the trace pairing

$$
B \wedge_{A} B \stackrel{\text { mult }}{\longrightarrow} B \stackrel{\text { tr }}{\longrightarrow} A
$$

which is clearly equivariant if we take the diagonal $G$-action on $B \wedge_{A} B$.

We also need to produce a coevaluation $\eta: A \longrightarrow B \wedge_{A} B$. Working in the derived category $\mathscr{D}_{A}$, this is done using the map $B \longrightarrow B \wedge_{A} B$ implicit in condition (BNG-2) of Definition 1.4.4 and splitting the multiplication map $B \wedge_{A} B \longrightarrow B$ which corresponds to projection onto the identity element component of $\prod_{\gamma \in G} B \cong F\left(G_{+}, B\right)$. The composition $\eta: A \longrightarrow B \longrightarrow B \wedge_{A} B$ can be viewed as the unique element of $\pi_{0}\left(B \wedge_{A} B\right)$ projecting to the element of $\left(\delta_{\gamma, 1}\right) \in \prod_{\gamma \in G} \pi_{0} B$, where

$$
\delta_{\alpha, \beta}= \begin{cases}1 & \text { if } \alpha=\beta \\ 0 & \text { otherwise }\end{cases}
$$

Now to show that $B$ is strongly self-dual with evaluation $\varepsilon$ and coevaluation $\eta$, we have to verify condition (b) of Theorem 1.3.3. We need to check that when $P=B=Q$, the compositions in the diagrams (1.3.1) are indeed the identity morphisms. 
For each $\gamma \in G$, there is a map of $A$-ring spectra

$$
\widetilde{\gamma}: B \wedge_{A} B \stackrel{\operatorname{id} \wedge \gamma}{\longrightarrow} B \wedge_{A} B \stackrel{\mu}{\longrightarrow} B .
$$

Recalling that as maps from $B$ to $B$,

$$
\iota \circ \operatorname{tr} \sim \sum_{\gamma \in G} \gamma,
$$

we find that the composition in (1.3.1a) is

$$
\sum_{\gamma \in G} \widetilde{\gamma} \circ(\mathrm{id} \wedge \text { mult }) \circ(\eta \wedge \mathrm{id}): B \cong A \wedge_{A} B \longrightarrow B .
$$

Since each $\widetilde{\gamma}$ is a $B$-bimodule morphism and because of the way $\eta$ was characterized in terms of its projections under the $\widetilde{\gamma}$, this composition is homotopic to

$$
\sum_{\gamma \in G} \delta_{\gamma, 1} \gamma=\mathrm{id}
$$

A similar discussion applies to the composition in (1.3.1b).

Remark 1.4.12. In general, in distinction to part (b) of Theorem 1.1.5, the trace map $\operatorname{tr}=\operatorname{tr}_{B / A}$ need not induce an epimorphism $\operatorname{tr}_{*}: B_{*} \longrightarrow A_{*}$. For example, in the case of $K U / K O$ discussed in Example 1.4.9, the trace map tr agrees with the realification map and $\operatorname{tr}_{*}(u)=\eta^{2}$. To see this, note that we are dealing with $K O$-module maps $K U \longrightarrow K O$. Then by the self-duality of the $K O$-module $K U$,

$$
\pi_{0} F_{K O}(K U, K O) \cong \pi_{0} F_{K O}(K O, K U) \cong \pi_{0} K U \cong \mathbb{Z} .
$$

This means that elements of $\mathscr{D}_{K O}(K U, K O) \cong \pi_{0} F_{K O}(K U, K O)$ are detected by their induced effect on the free abelian homotopy groups $\pi_{4 n} K U \longrightarrow \pi_{4 n} K O$ for $n \in \mathbb{Z}$, and using the $K O_{*}$-module structure we find that this is determined by the homomorphism $\pi_{0} K U \longrightarrow \pi_{0} K O$. Thus it suffices to know the standard fact that the realification map $K U \longrightarrow K O$ induces $2: \pi_{0} K U \longrightarrow \pi_{0} K O$.

When the order of the Galois group $G$ is invertible in $A_{*}$, such anomalies do not occur.

Proposition 1.4.13. Let $A$ and $B$ be commutative $\mathbb{S}$-algebras and let $A \longrightarrow B$ be a weak $G$-Galois extension for which $A_{*}$ is a $\mathbb{Z}[1 /|G|]$-algebra. Then the following hold:

(a) The unit $A \longrightarrow B$ induces a monomorphism $A_{*} \longrightarrow B_{*}$.

(b) The unit $A \longrightarrow B$ and trace $\operatorname{tr}: B \longrightarrow A$ compose to an equivalence on $A$. Hence an $A$-module $M$ is a retract of $B \wedge_{A} M$. In particular, $B$ is faithful.

Proof. (a) There is a spectral sequence

$$
\mathrm{E}_{2}^{s, t}=\mathrm{H}^{s}\left(G ; B_{t}\right) \Longrightarrow\left(B^{\mathrm{h} G}\right)_{t-s} .
$$

Under the above hypotheses, the $\mathrm{E}_{2}$-term is concentrated in the zero-line where $\mathrm{E}_{2}^{0, t}=\left(B_{t}\right)^{G}$. Hence on homotopy groups, the unit induces the inclusion of the fixed points of $B_{*}$.

(b) In the diagram of (1.4.3), precomposition of the top row with the unit $\iota: A \longrightarrow B$ induces an equivalence $\operatorname{tr} \iota: A \longrightarrow A$. Thus the trace and unit are split. It follows that an $A$-module $M$ is a retract of $B \wedge_{A} M$.

Part (b) of the last proposition implies the following. 
Corollary 1.4.14. Let $A$ and $B$ be commutative $\mathbb{S}$-algebras and let $B / A$ be a weak $G$-Galois extension for which $A_{*}$ is a $\mathbb{Z}[1 /|G|]$-algebra. Then $B / A$ is a $G$-Galois extension.

The following example is straightforward to verify. Let $p$ be an odd prime. The Johnson-Wilson spectrum $E(1)$ agrees with the Adams summand of $K U_{(p)}$. Passing to the $p$-completions, there is an action of $C_{p-1}$ on $\left(K U_{p}\right)$ (see [4, Theorem 9.2], $[13, \S 7])$ that is easily seen to turn $K U_{p}$ into a weak Galois extension of $E(1)_{p}$. We can use Corollary 1.4 .14 to obtain

Example 1.4.15. $K U_{p} / E(1)_{p}$ is a $C_{p-1}$-Galois extension.

There is analogue of Proposition 1.1.10 namely

Proposition 1.4.16. Let $B / A$ and $C / A$ be $G$-Galois extensions and let $\varphi: B \longrightarrow C$ be a morphism of A-algebras which commutes with the action of $G$. Then $\varphi$ is a weak equivalence.

Proof. By the faithfulness of $B$ and $C$, it suffices to check this for the morphism $\widetilde{\varphi}=\operatorname{id} \wedge \operatorname{id} \wedge \varphi$ between the $G$-Galois extensions $B \wedge_{A} C \wedge_{A} B / B \wedge_{A} C$ and $B \wedge_{A}$ $C \wedge_{A} C / B \wedge_{A} C$. But then

$$
B \wedge_{A} C \wedge_{A} B \sim \prod_{\gamma \in G} B \wedge_{A} C \sim B \wedge_{A} C \wedge_{A} C .
$$

Now by construction, the map id $\wedge \Theta$ of Definition 1.4.4(BNG-2) is $B \wedge C$-linear, hence $\widetilde{\varphi}$ induces a $B_{*}^{A} C$-algebra homomorphism

$$
\widetilde{\varphi}_{*}: \prod_{\gamma \in G} B_{*}^{A} C \longrightarrow \prod_{\gamma \in G} B_{*}^{A} C
$$

which is $G$-equivariant, and by Proposition 1.1.10, this is an isomorphism.

\section{Part 2. From algebraic Galois extensions to brave new Galois extensions}

\subsection{Topological Realization of algebraic Galois extensions}

Let $A$ be a commutative $\mathbb{S}$-algebra and let $G$ be a finite group. Also recall Proposition 1.1 .10

Theorem 2.1.1. If $B_{*}$ is a $G$-Galois extension of $A_{*}$, then there is a commutative $A$-ring spectrum $B$ realizing $B_{*}$ as $\pi_{*} B$ and a homotopy action of $G$ on $B$ by morphisms of $A$-ring spectra which induce the action of $G$ on $B_{*}$. Furthermore, if $C_{*}$ is also a $G$-Galois extension of $A_{*}$ and there is a $G$-isomorphism $\Phi: B_{*} \longrightarrow C_{*}$ of $A_{*}$-algebras, then there is a map of $A$-ring spectra $\varphi: B \longrightarrow C$ which induces $\Phi$. It is $G$-equivariant up to homotopy.

Proof. By Theorem 1.1.4(d), $B_{*}$ is a finitely generated projective $A_{*}$-module, so we can realize $B_{*}$ as the image of an idempotent $e: \bigoplus_{i=1}^{n} \Sigma^{m_{i}} A_{*} \longrightarrow \bigoplus_{i=1}^{n} \Sigma^{m_{i}} A_{*}$. We can model the map $e$ on a wedge of suspensions of $A$. Therefore the mapping telescope of

$$
\bigvee \Sigma^{m_{i}} A \stackrel{e}{\rightarrow} \bigvee \Sigma^{m_{i}} A \stackrel{e}{\rightarrow} \cdots
$$

gives rise to an $A$-module spectrum $B$ with $\pi_{*} B \cong B_{*}$. 
The Künneth spectral sequence

$$
\mathrm{E}_{p, q}^{2}=\operatorname{Tor}_{p, q}^{A_{*}}\left(B_{*}, B_{*}\right) \Longrightarrow B_{p+q}^{A} B
$$

of [11] collapses to give

$$
B_{*}^{A} B=B_{*} \otimes_{A_{*}} B_{*} .
$$

More generally, for each $n \geqslant 2$,

$$
\pi_{*} B^{(n)} \cong \overbrace{B_{*}^{A} B \otimes_{B_{*}} B_{*}^{A} B \otimes_{B_{*}} \cdots \otimes_{B_{*}} B_{*}^{A} B}^{n-1},
$$

where ()$^{(n)}$ denotes the $n$-fold smash product over $A$. This is projective, both as an $A_{*}$-module and as a $B_{*}$-module.

From [11, for each $A$-module $Y$ there is a universal coefficient spectral sequence

$$
\mathrm{E}_{2}^{p, q}=\operatorname{Ext}_{A_{*}}^{p, q}\left(\pi_{*} B^{(n)}, Y_{*}\right) \Longrightarrow Y_{A}^{p+q}\left(B^{(n)}\right) .
$$

By the projectivity of the first variable, this spectral sequence collapses to give

$$
Y_{A}^{*}\left(B^{(n)}\right) \cong \operatorname{Hom}_{A_{*}}^{*}\left(\pi_{*} B^{(n)}, Y_{*}\right) .
$$

The product on $B_{*}$ is an element of $\operatorname{Hom}_{A_{*}}\left(\pi_{*}\left(B \wedge_{A} B\right), B_{*}\right)$ which corresponds to a unique element of $B^{0}\left(B \wedge_{A} B\right)$. Since $B_{*}$ is a commutative $A_{*}$-algebra, this product on $B$ is homotopy associative, commutative and unital over $A$.

Similarly, the action of elements of $G$ on $B_{*}$ induces a homotopy action of $G$ on $B$ by morphisms of $A$-ring spectra.

Since $B_{*}$ is finitely generated and projective as an $A_{*}$-module, the relevant universal coefficient spectral sequence collapses, and for $m, k \in \mathbb{N}, n \in \mathbb{Z}$ there are isomorphisms

$$
\mathscr{D}_{A}\left(B^{(k)}, \Sigma^{n} C^{(m)}\right) \stackrel{\cong}{\longrightarrow} \operatorname{Hom}_{A_{*}}^{n}\left(\left(B_{*}\right)^{\otimes k},\left(C_{*}\right)^{\otimes m}\right),
$$

where ()$^{(k)}$ denotes the $k$-fold smash product over $A$.

Let $\operatorname{Hom}_{A_{*}-\text { alg }}\left(B_{*}, C_{*}\right)$ denote the set of $G$-equivariant $A_{*}$-algebra maps from $B_{*}$ to $C_{*}$, and let $\operatorname{Hom}_{A_{*}-\operatorname{alg}}\left(B_{*}, C_{*}\right)^{G} \subseteq \operatorname{Hom}_{A_{*}-\operatorname{alg}}\left(B_{*}, C_{*}\right)$ be the subset of $G$ equivariant maps. The latter can be written as an iterated equalizer: first we obtain $\operatorname{Hom}_{A_{*}-\operatorname{alg}}\left(B_{*}, C_{*}\right)$ as the equalizer

$$
\operatorname{Hom}_{A_{*}-\operatorname{alg}}\left(B_{*}, C_{*}\right) \longrightarrow \operatorname{Hom}_{A_{*}}\left(B_{*}, C_{*}\right) \Longrightarrow \operatorname{Hom}_{A_{*}}\left(B_{*} \otimes_{A_{*}} B_{*}, C_{*}\right) .
$$

The projectivity of $B_{*}$ over $A_{*}$ therefore gives that $\operatorname{Hom}_{A_{*}-\operatorname{alg}}\left(B_{*}, C_{*}\right)$ is the same as the homotopy classes of maps of $A$-ring spectra from $B$ to $C,[B, C]_{A \text {-ring. }}$. Similarly, as $G$ is finite we know that $\left(G_{+} \wedge B\right)_{*}$ is finitely generated projective over $A_{*}$, and we obtain that the homotopy classes of $G$-equivariant maps of $A$ ring spectra, $[B, C]_{A-\text { ring }}^{G}$, are the same as $\operatorname{Hom}_{A_{*}-\text { alg }}\left(B_{*}, C_{*}\right)^{G}$. The map $\Phi$ is an element of the latter; therefore there is a realization $\varphi: B \longrightarrow C$, which is homotopy $G$-equivariant and a map of $A$-ring spectra. 


\subsection{Realizing Galois extensions}

In this section we will assume that the following two conditions hold.

(GE-1) $A$ is a commutative $\mathbb{S}$-algebra, and $B$ is a commutative $A$-ring spectrum. There is a homotopy action of the finite group $G$ on $B$ viewed as an $A$ module, i.e., there is a homomorphism of monoids $G \longrightarrow \mathscr{D}_{A}(B, B)$ which is in fact an action by automorphisms of $B$ as an $A$-ring spectrum, i.e., the homomorphism $G \longrightarrow \mathscr{D}_{A}(B, B)$ is compatible with the product $\mu: B \wedge_{A}$ $B \longrightarrow B$ in $\mathscr{D}_{A}$.

(GE-2) The $A_{*}$-algebra $B_{*}$ is a $G$-Galois extension with respect to the induced action of $G$, thus the action of $G$ on $B_{*}$ is effective, i.e., $G \leqslant \operatorname{Aut}_{A_{*}}\left(B_{*}\right)$, and satisfies the axioms of a Galois action (see Definition 1.1.1).

For instance, these conditions are satisfied when we start with a situation as in Theorem 2.1.1.

Proposition 2.2.1. As an $A_{*}$-algebra,

$$
B_{*}^{A} B=\pi_{*}\left(B \wedge_{A} B\right) \cong \prod_{\gamma \in G} B_{*},
$$

where the map is induced by that of (1.4.1). Hence $B_{*}^{A} B$ is an étale $B_{*}$-algebra.

Proof. Recalling (2.1.3), we see that

$$
B_{*}^{A} B \cong B_{*} \otimes_{A_{*}} B_{*} .
$$

As the edge homomorphism in the Künneth spectral sequence (2.1.2) is multiplicative, this is an isomorphism of $A_{*}$-algebras. Since $B_{*}$ is étale over $A_{*}, B_{*} \otimes_{A_{*}} B_{*}$ is étale over $B_{*}$. Hence $B_{*}^{A} B$ is étale over $B_{*}$.

Corollary 2.2.2. For any $B_{*}^{A} B$-bimodule $M_{*}$ and $B_{*}^{A} B$-module $N_{*}$, the Hochschild cohomology and the $\Gamma$-cohomology of $B_{*}^{A} B$ relative to $B_{*}$ vanish, i.e.,

$$
\mathrm{HH}^{* *}\left(B_{*}^{A} B \mid B_{*} ; M_{*}\right) \cong M_{*} \quad \text { and } \mathrm{H}^{* *}\left(B_{*}^{A} B \mid B_{*} ; N_{*}\right)=0 .
$$

Proposition 2.2.3. Assume that $A, B, G$ satisfy conditions (GE-1) and (GE-2). Then the A-ring spectrum structure on $B$ has a refinement to a commutative $A$ algebra structure which is unique up to contractible choice.

Proof. We will use adaptations of the obstruction theory of Robinson to the relative case. This approach has been set up to establish the existence of $E_{\infty}$ structures (or equivalently commutative $\mathbb{S}$-algebra structures) on spectra. Our aim is to establish commutative $A$-algebra structures on a homotopy commutative $A$-ring spectrum $B$.

The geometric nature of Robinson's obstruction groups as described in [23, §5] ensures that the obstructions for imposing a commutative $A$-algebra structure on $B$ live in $\Gamma$-cohomology $\mathrm{H}^{* *}\left(B_{*}^{A} B \mid B_{*} ; B_{*}\right)$ of $\left(B \wedge_{A} B\right)_{*}$ relative to $B_{*}$. Using the notation of [23, Definition 5.3], an $n$-stage for such a structure corresponds to action maps

$$
\mu_{m}: \nabla^{n} \mathcal{T}_{m} \ltimes_{\Sigma_{m}} \overbrace{B \wedge_{A} \cdots \wedge_{A} B}^{m} \longrightarrow B
$$

for $m \leqslant n$ and certain compositions. As we have assumed that $A_{*} \longrightarrow B_{*}$ is $G$ Galois, we find that $B_{*}$ is $A_{*}$-projective; hence one obtains universal coefficient and Künneth isomorphisms which identify $B_{A}^{*}\left(B \wedge_{A} B\right)$ with $\operatorname{Hom}_{B_{*}}\left(B_{*} \otimes_{A_{*}} B_{*}, B_{*}\right)$. 
The result is now immediate using the modified obstruction theory of Robinson [23, 24] and general properties of $\Gamma$-cohomology established by Robinson and Whitehouse [25] for $A \longrightarrow B$, because $B_{*} \otimes_{A_{*}} B_{*}$ is étale over $B_{*}$ and hence the obstruction groups vanish.

Now by making use of results of Robinson and Whitehouse [23, 24, 25, and Goerss and Hopkins [13, we obtain

Theorem 2.2.4. Assume that A, B, G satisfy conditions (GE-1) and (GE-2). Then the following hold:

(a) Each element $\gamma \in G$ induces a morphism of $A$-algebras from $B$ to $B$ which is unique up to contractible choice.

(b) The morphisms of part (a) combine to give an action of $G$ on $B$ by $A$ algebra automorphisms.

(c) Suppose that A, C, G also satisfy the conditions of (GE-1) and (GE-2), thus there is a unique A-algebra structure on $C$ as in (a). If $\widetilde{\varphi}: B \longrightarrow C$ is a map of A-ring spectra which is $G$-equivariant up to homotopy, then there are commutative A-algebras $B^{\prime \prime}$ and $C^{\prime \prime}$ together with weak equivalences $B \sim$ $B^{\prime \prime}$ and $C \sim C^{\prime \prime}$. These weak equivalences are zigzags of weak equivalences of commutative A-algebras which are $G$-equivariant up to homotopy. There is a map of commutative A-algebras $\bar{\varphi}: B^{\prime \prime} \rightarrow C^{\prime \prime}$ which is strictly $G$ equivariant and which induces $\widetilde{\varphi}$.

Proof. For (a) and (b), the desired result comes from the triviality of the spectral sequence for the homotopy of the derived space of commutative $A$-algebra self-maps of $B$ based at any choice of map in the $A$-algebra homotopy class of an element of $G$ realized as an $A$-algebra morphism. More precisely, we use the generalization of [13. Theorem 4.5] to the setting of $A$-algebras and take $E=X=Y=B$. Note that the description of $B$ as in (2.1.1) ensures that $B$ satisfies the Adams condition required in [13, Definition 3.1].

Now we make a modification of [13, Definition 3.2], using as $\mathcal{P}$ the set of spectra consisting of the $A$-sphere $S_{A}, B$, their suspensions and finite wedges of these. As $B_{*}$ is finitely generated $A_{*}$-projective, we have a universal coefficient isomorphism.

The second quadrant spectral sequence converging to the homotopy groups of the derived space of self-maps of $B$ in the category of $E_{\infty}$-algebras in $A$-modules, $\operatorname{map}_{E_{\infty}-A \text {-alg }}(B, B)$, looks as follows. The $\mathrm{E}_{2}$-term is

$$
\mathrm{E}_{2}^{s, t}= \begin{cases}\operatorname{Hom}_{B_{*}-\operatorname{alg}}\left(B_{*}^{A} B, B_{*}\right) & \text { for }(s, t)=(0,0), \\ \operatorname{Der}_{B_{*}}^{s}\left(B_{*}^{A} B, \Omega^{t} B_{*}\right) & \text { for } t>0,\end{cases}
$$

where $\operatorname{Der}_{B_{*}}^{s}\left(B_{*}^{A} B, \Omega^{t} B_{*}\right)$ denotes the $s$-th derived functor of derivations into the $t$-th shift of $B_{*}$ and $\operatorname{Hom}_{B_{*} \text {-alg }}\left(B_{*}^{A} B, B_{*}\right)$ are the morphisms of $B_{*}$-algebras from $B_{*}^{A} B \cong B_{*} \otimes_{A_{*}} B_{*}$ to $B_{*}$. For $s>0$ we know that $\operatorname{Der}_{B_{*}}^{s}\left(B_{*}^{A} B, \Omega^{t} B_{*}\right)$ vanishes since $B_{*}^{A} B$ is étale over $B_{*}$. The reader might wish to use the comparison result of [6] to see that. In this case it provides an isomorphism between $\operatorname{Der}_{B_{*}}^{s}\left(B_{*}^{A} B, \Omega^{-t} B_{*}\right)$ and $\mathrm{H} \Gamma^{s, t}\left(B_{*}^{A} B \mid B_{*} ; B_{*}\right)$. There should be a direct proof as well. As a basepoint for the derived mapping space $\operatorname{map}_{E_{\infty}-A \text {-alg }}(B, B)$, we take the identity map of $B$.

Since $B_{*}$ is $G$-Galois over $A_{*}$, each group element $\gamma \in G$ gives rise to an element in the morphism set $\operatorname{Hom}_{B_{*}-\operatorname{alg}}\left(B_{*}^{A} B, B_{*}\right)$ which sends $b_{1} \otimes b_{2}$ to $b_{1}{ }^{\gamma} b_{2}$. 
Using the partially invisible corollary [13, Corollary to Theorem 4.5, analogous to 4.4], we see that the vanishing of the obstruction groups $\operatorname{Der}_{B_{*}}^{s}\left(B_{*}^{A} B, \Omega^{s} B_{*}\right)$ and $\operatorname{Der}_{B_{*}}^{s+1}\left(B_{*}^{A} B, \Omega^{s} B_{*}\right)$ implies that the Hurewicz map

$$
\pi_{0}\left(\operatorname{map}_{E_{\infty}-A \text {-alg }}(B, B)\right) \longrightarrow \operatorname{Hom}_{B_{*}-\text { alg }}\left(B_{*}^{A} B, B_{*}\right)
$$

is a bijection, hence each component is labelled by an element of the latter. (The condition for surjectivity is stated in [13, Theorem 4.5].) Therefore the derived mapping space $\operatorname{map}_{E_{\infty}-A \text {-alg }}(B, B)$ has contractible components, because for étale algebras like $B_{*}^{A} B$ Gamma cohomology vanishes with arbitrary coefficients. In addition we see that the group $G$ is a submonoid of $\pi_{0}\left(\operatorname{map}_{E_{\infty}-A \text {-alg }}(B, B)\right)$, in particular every $\gamma \in G$ gives a self-map of $B$ in the homotopy category of $E_{\infty}-A$ algebras. In the terminology of [10] (or of [8, Definition 2.1]) the diagram category consisting of the group viewed as a one-object category gives rise to an $h_{\infty}$-diagram: Let $X(0)$ be $B$ and for every $\gamma \in G$ we obtain a self-map $X(0, \gamma)$ of $B$. The pathcomponent of the image of $\gamma$ is contractible. Using [8, Theorem 2.2] we can strictify this diagram such that there is a weakly equivalent $E_{\infty}-A$-algebra $B^{\prime}$ with a strict $G$-action.

For part (c) the arguments are a little bit more involved. As we saw, we can realize $A$-algebras $B^{\prime}$ and $C^{\prime}$ with actual $G$-actions, such that $B^{\prime}$ is weakly equivalent to $B$ and $C^{\prime}$ is weakly equivalent to $C$ via maps of $E_{\infty}-A$-algebras. As $\widetilde{\varphi}$ was a map of $A$-ring spectra, it gives rise to a map $\varphi$ of homotopy $A$-ring spectra from $B^{\prime}$ to $C^{\prime}$ which is $G$-equivariant up to homotopy. In particular, the map on $C$-homology, $C_{*}(\varphi)$, is a map of commutative $C_{*}$-algebras. As $B_{*}$ is étale over $A_{*}$ a base-change argument implies that $C_{*}^{A} B \cong C_{*} \otimes_{A_{*}} B_{*}$ is $C_{*}$-étale.

Using the Hurewicz argument again, $C_{*}(\varphi)$ gives rise to a map $\varphi^{\prime}$ of $E_{\infty}-A$ algebras from $B^{\prime}$ to $C^{\prime}$ which is still $G$-equivariant up to homotopy.

We claim that the following is an $h_{\infty}$-diagram: take $X(0)=B^{\prime}$ and $X(1)=C^{\prime}$ as vertices, and for every group element $\gamma \in G$ we get morphisms $X(\gamma, 0)$ from $X(0)$ to itself and $X(\gamma, 1)$ on $X(1)$. We place the map $\varphi^{\prime}: B^{\prime} \longrightarrow C^{\prime}$ in the diagram as a connection between $X(0)$ and $X(1)$ :

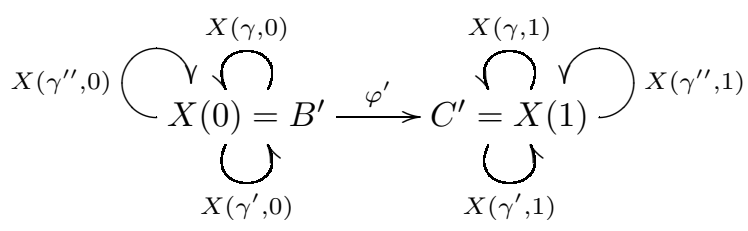

For the element in $\pi_{0}\left(\operatorname{map}_{E_{\infty}-A \text {-alg }}(B, C)\right)$ corresponding to $C_{*}(\varphi)$ we get an arrow from $X(0)$ to $X(1)$ in the homotopy category of $E_{\infty}$-A-algebras. As every component in $\operatorname{map}_{E_{\infty}-A \text {-alg }}(B, C)$ is contractible, one can strictify $X$ to get a weakly equivalent diagram $\bar{X}$. By construction, these equivalences are maps of commutative $A$-algebras which are $G$-equivariant up to homotopy. The arrow $\bar{\varphi}$ from $\bar{X}(0)$ to $\bar{X}(1)$ is $G$-equivariant by construction and is a weak equivalence due to three-out-of-four.

Proposition 2.2.5. Assume that $A, B, G$ satisfy conditions (GE-1) and (GE-2). Then the trace map $\operatorname{tr}: B \longrightarrow A$ induces a surjection $\operatorname{tr}_{*}: B_{*} \longrightarrow A_{*}$. 
Proof. As $B_{*} / A_{*}$ is $G$-Galois, the trace map is an epimorphism by Theorem 1.1.5(b).

Corollary 2.2.6. The unit $A \longrightarrow B$ is split. Hence, $B$ is faithful as an $A$-module.

Proof. Let $b: A \longrightarrow B$ be an element of $B_{0}=\pi_{0} B$ for which $\operatorname{tr}_{*}(b)=1$. Then the composition

$$
B \sim A \wedge_{A} B \stackrel{b \wedge \text { id }}{\longrightarrow} B \wedge_{A} B \stackrel{\text { mult }}{\longrightarrow} B \stackrel{\text { tr }}{\longrightarrow} A
$$

splits the unit. Hence for any $A$-module $M, M$ is a retract of $B \wedge_{A} M$, and so $B$ is faithful.

Now we can state and prove the main result of this section.

Theorem 2.2.7. Assume that $A, B, G$ satisfy conditions (GE-1), (GE-2). Then $B / A$ is a $G$-Galois extension.

Proof. Without loss of generality we can replace $B$ by a cofibrant commutative $A$-algebra: there is a functorial cofibrant replacement functor $Q(-)$ (see [11, VII, $\S \S 4,5])$; therefore $Q(B)$ inherits the $G$-action from $B$. As $\pi_{*} Q(B) \cong B_{*}$ is still $A_{*}$-projective, $Q(B)$ is unramified. In the following we write $B$ instead of $Q(B)$.

We use the homotopy fixed point spectral sequence

$$
\mathrm{E}_{2}^{s, t}=\mathrm{H}^{s}\left(G ; B_{t}\right) \Longrightarrow\left(B^{\mathrm{h} G}\right)_{t-s}
$$

to ensure that $B$ has the correct homotopy fixed points with respect to the $G$-action. We suppress the internal grading to ease notation.

Now by Proposition 1.1.8

$$
\mathrm{E}_{2}^{*}=\mathrm{E}_{2}^{0}=\operatorname{Hom}_{A_{*} G}\left(A_{*}, B_{*}\right) \cong\left(B_{*}\right)^{G}=A_{*} .
$$

Therefore $\pi_{*}\left(B^{\mathrm{h} G}\right) \cong A_{*}$, and so $B^{\mathrm{h} G} \sim A$.

Example 2.2.8. For $n \geqslant 1$ and $\zeta_{n^{\ell}}$ a primitive $n^{\ell}$-th root of unity, we may consider the ring $\mathbb{Z}\left[1 / n, \zeta_{n^{\ell}}\right] \subseteq \mathbb{C}$. The prime factors of the discriminant of $\mathbb{Z}\left[1 / n, \zeta_{n^{\ell}}\right]$ over $\mathbb{Z}[1 / n]$ are known to divide $n$, so $\mathbb{Z}\left[1 / n, \zeta_{n^{\ell}}\right]$ is unramified over $\mathbb{Z}[1 / n]$. Then $\mathbb{Z}\left[1 / n, \zeta_{n^{\ell}}\right] / \mathbb{Z}[1 / n]$ is a $\left(\mathbb{Z} / n^{\ell}\right)^{\times}$-Galois extension, and there is an isomorphism of $\mathbb{Z}\left[1 / n, \zeta_{n^{\ell}}\right]$-algebras

$$
\mathbb{Z}\left[1 / n, \zeta_{n^{\ell}}\right] \otimes_{\mathbb{Z}[1 / n]} \mathbb{Z}\left[1 / n, \zeta_{n^{\ell}}\right] \cong \prod_{\gamma \in\left(\mathbb{Z} / n^{\ell}\right)^{\times}} \mathbb{Z}\left[1 / n, \zeta_{n^{\ell}}\right] .
$$

For any commutative $\mathbb{S}$-algebra $A$, by Theorem 2.1.1 we can give $B=A \mathbb{Z}\left[1 / n, \zeta_{n^{\ell}}\right]$ the structure of a commutative $A$-ring spectrum. By applying Theorem 2.2.4, we see that the ring $A_{*} \otimes \mathbb{Z}\left[1 / n, \zeta_{n^{\ell}}\right]$ can be realized as the homotopy ring of a commutative $A[1 / n]$-algebra. Thus we find that $B / A$ is a $\left(\mathbb{Z} / n^{\ell}\right)^{\times}$-Galois extension. This gives a different approach to results of 27.

Example 2.2.9. For a prime $p$, let $E_{n}$ denote the 2-periodic Lubin-Tate spectrum whose homotopy ring is

$$
\left(E_{n}\right)_{*}=\mathrm{WF}_{p^{n}}\left[\left[u_{1}, \ldots, u_{n-1}\right]\right]\left[u, u^{-1}\right],
$$

where the $u_{i}$ are of degree zero and $u$ is an element of degree -2 . This is known to be an algebra over the $I_{n}$-adic completion $\widehat{E(n)}$ of the Johnson-Wilson spectrum $E(n)$ (see [4] for a proof that $\widehat{E(n)}$ is commutative). On coefficients, the ring map from $\widehat{E(n)})_{*}$ to $\left(E_{n}\right)_{*}$ is determined by $v_{i} \mapsto u_{i} u^{1-p^{i}}$. Then $E_{n} / \widehat{E(n)}$ is a $C_{n} \ltimes \mathbb{F}_{p^{n}}^{\times}$-Galois extension. 


\subsection{Topological Harrison groups}

As we want to compare algebraic Galois extensions to topological ones, we propose the following definitions of Harrison sets for a commutative $\mathbb{S}$-algebra $A$ and a finite group $G$.

Definition 2.3.1. We call two $G$-Galois extensions of $A, B^{\prime}$ and $B^{\prime \prime}$, weakly Harrison equivalent if there are commutative $A$-algebras with a homotopy $G$-action $\left(B_{i}\right)_{i=1}^{n}$ and commutative $A$-algebras with strict $G$-action $\left(B_{i}^{\prime}\right)_{i=1}^{n-1}$ with weak equivalences of $A$-algebras $\varepsilon_{i}, \rho_{i}$ which are homotopy $G$-equivariant, as in the following diagram:

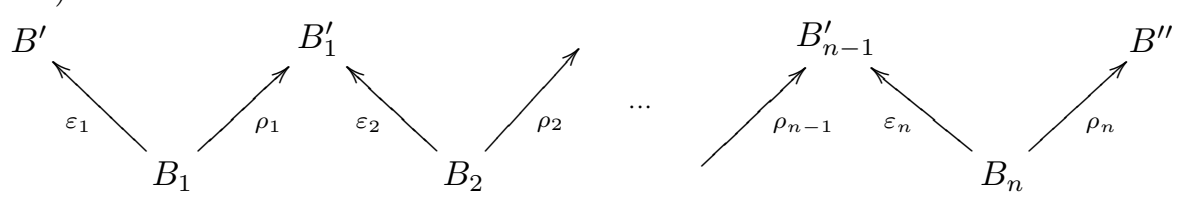

We denote the set of such equivalence classes by $\operatorname{Har}^{w}(A, G)$.

Definition 2.3.2. We call two $G$-Galois extensions of $A, B^{\prime}$ and $B^{\prime \prime}$ Harrison equivalent if there are commutative $A$-algebras with a strict $G$-action $\left(B_{i}\right)_{i=1}^{n}$ and commutative $A$-algebras with strict $G$-action $\left(B_{i}^{\prime}\right)_{i=1}^{n-1}$ with weak equivalences of $A$ algebras $\varepsilon_{i}, \rho_{i}$ which are $G$-equivariant and which fit diagram (2.3.1). Such equivalence classes are denoted by $\operatorname{Har}(A, G)$.

Remark 2.3.3.

- The weak equivalence notion comes out of our realization result. We propose the definition of $\operatorname{Har}(A, G)$ as a compromise. It is strong enough to prove structural results. Note that there is an obvious map

$$
\operatorname{Har}(A, G) \longrightarrow \operatorname{Har}^{w}(A, G) .
$$

- A map in the other direction would require us to replace $A$-algebras with homotopy $G$-actions by $A$-algebras with strict $G$-action. This means that we have to compare the mapping space of $G$-equivariant maps from $E G$ to the mapping space of $A$-algebra endomorphisms of an algebra spectrum $B$ with the mapping space of $G$-equivariant maps from a point to that endomorphism space. This boils down to proving the Sullivan conjecture for the endomorphism space of $A$-algebra maps on $B$.

Theorem 2.3.4. The constructions of the last section produce a map

$$
\operatorname{Real}_{G}: \operatorname{Har}\left(A_{*}, G\right) \longrightarrow \operatorname{Har}^{w}(A, G) .
$$

Proof. We must show that the topological realization of an algebraic map $\Phi: B_{*} \longrightarrow$ $C_{*}$ of $G$-Galois extensions is unique up to $G$-equivariant homotopy. Assume there are two such realizations, $\varphi$ and $\psi$, of $\Phi$, which are maps of commutative $A$-algebras. The connected components of $\operatorname{map}_{E_{\infty}-A \text {-alg }}(B, C)$ are labelled by the elements of $\operatorname{Hom}_{A_{*}-\operatorname{alg}}\left(B_{*}, C_{*}\right)$. Thus $\varphi$ and $\psi$ are in the same path-component, and a path between these gives a homotopy $H$. The group action of $G$ preserves the components. Therefore any element $g \in G$ applied to $\varphi$ and $\psi$ is again in that component, and so is $g$ applied to $H$. We connect $\varphi$ with $g \varphi g^{-1}$, and similarly $\psi$ and $H$ with 
their conjugates. Schematically this yields the following diagram:

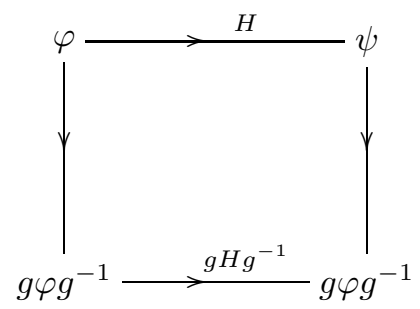

As the components are contractible, we can fill in the rectangle whose boundary is made out of homotopies. This proves that $H$ is $G$-equivariant up to homotopy.

Proposition 2.3.5. The Harrison set $\operatorname{Har}(A, G)$ is natural in $G$. When $G$ is an abelian group, then $\operatorname{Har}(A, G)$ is an abelian group as well.

Proof. We give a translation of Greither's proof from [16] to the topological setting. Given a group homomorphism $\varphi: G \longrightarrow H$, for a $G$-Galois extension $B / A$ we define

$$
\varphi_{*} B=F\left(H_{+}, B\right)^{\mathrm{h} G}=F\left(E G_{+}, F\left(H_{+}, B\right)\right)^{G},
$$

the homotopy fixed points of the function spectrum $F\left(H_{+}, B\right)$ with the action coming from the left $G$-action on $H$ and $B$. Then $\varphi_{*} B$ has a natural $H$-action induced from the right action of $H$ on itself.

Choose a free contractible left $G$-space $E G$ and a free contractible left $H$-space $E H$ and also write $E^{\prime} H$ for $E H$ with the trivial $H$-action but also viewed as a left $G$-space through the homomorphism $\varphi$. We also view $H$ as a left $G$-space via the homomorphism $\varphi$ and as a left $H$-space by inverse right multiplication. There is a left $G$-action on $H \times_{H} E H$ induced from the action on the $H$ factor, and the isomorphism

$$
H \times_{H} E H \cong E^{\prime} H ; \quad[\eta, x] \longleftrightarrow \eta x
$$

is $G$-equivariant.

Consider a chain of homomorphisms of groups $G \stackrel{\varphi}{\rightarrow} H \stackrel{\psi}{\longrightarrow} K$. We have to prove that

$$
\psi_{*}\left(\varphi_{*} B\right) \sim(\psi \circ \varphi)_{*} B .
$$

To this end we have to compare $F\left(K_{+}, F\left(H_{+}, B\right)^{\mathrm{h} G}\right)^{\mathrm{h} H}$ with $F\left(K_{+}^{\prime}, B\right)^{\mathrm{h} G}$, where $K^{\prime}$ denotes $K$ with the left action of $G$ via $\psi \circ \varphi$. There is a chain of equivalences

$$
\begin{aligned}
F\left(K_{+}, F\left(H_{+}, B\right)^{\mathrm{h} G}\right)^{\mathrm{h} H} & =F\left(E H_{+}, F\left(K_{+}, F\left(E G_{+}, F\left(H_{+}, B\right)\right)^{G}\right)\right)^{H} \\
& \cong F\left(E G \times(H \times E H) \times_{H} K_{+}, B\right)^{G} \\
& \cong F\left(E G \times E^{\prime} H \times K_{+}^{\prime}, B\right)^{G} \\
& \simeq F\left(E G \times K_{+}^{\prime}, B\right)^{G} \\
& \cong F\left(E G_{+}, F\left(K_{+}^{\prime}, B\right)\right)^{G}=F\left(K_{+}^{\prime}, B\right)^{\mathrm{h} G} .
\end{aligned}
$$

We consider $B \wedge_{A} \varphi_{*} B$. The left-hand factor of $B$ has a trivial $G$-action, and $B$ is strongly dualizable. Hence it is equivalent to $\varphi_{*}\left(B \wedge_{A} B\right)$ by the following chain 
of identifications:

$$
\begin{aligned}
B \wedge_{A} \varphi_{*} B & =B \wedge_{A} F\left(E G_{+}, F\left(H_{+}, B\right)\right)^{G} \\
& \cong B \wedge_{A} F\left((E G \times H)_{+}, B\right)^{G} \\
& \stackrel{\sim}{\longrightarrow} F\left((E G \times H)_{+}, B \wedge_{A} B\right)^{G} \\
& \cong F\left(E G_{+}, F\left(H_{+}, B \wedge_{A} B\right)\right)^{G} \\
& =\varphi_{*}\left(B \wedge_{A} B\right) .
\end{aligned}
$$

Using Proposition 1.4.5 (c) (or results of [26]) it is enough to check that $B \wedge_{A} \varphi_{*} B$ is an $H$-Galois extension of $B$.

In order to check this, we consider the two natural inclusions of the trivial group

$$
G \stackrel{i}{\longleftarrow} e \stackrel{j}{\hookrightarrow} H
$$

It is obvious that $i_{*} B \cong \prod_{G} B$ and $j_{*} B \cong \prod_{H} B$. Using naturality (2.3.3) and $\varphi \circ i=j$, we obtain

$$
\varphi_{*}\left(B \wedge_{A} B\right) \cong \varphi_{*}\left(\prod_{G} B\right) \cong \varphi_{*}\left(i_{*} B\right) \stackrel{\sim}{\longleftarrow} j_{*} B \cong \prod_{H} B
$$

Therefore $\varphi_{*} B$ is unramified with respect to the $H$-action, and its $H$-homotopy fixed points agree with $A$.

If we consider abelian Galois extensions, then the source and target in (2.3.2) have abelian group structures. On the algebraic side, the map induced by the abelian multiplication $\mu: G \times G \longrightarrow G$ is a homomorphism which sends two $G$ Galois extensions $B_{*} / A_{*}$ and $C_{*} / A_{*}$ to $\mu_{*}\left(B_{*} \otimes_{A_{*}} C_{*}\right) / A_{*}$. Since the multiplication homomorphism is surjective, there is a short exact sequence

$$
0 \rightarrow K=\operatorname{ker} \mu \longrightarrow G \times G \stackrel{\mu}{\longrightarrow} G \rightarrow 0,
$$

and so using Harrison's formula [17, p. 3] we obtain

$$
\mu_{*}\left(B_{*} \otimes_{A_{*}} C_{*}\right)=\left(B_{*} \otimes_{A_{*}} C_{*}\right)^{K} .
$$

Mimicking this in the geometric situation we set $B \cdot C=\left(B \wedge_{A} C\right)^{\mathrm{h} K}$ for any abelian $G$-Galois extensions $B$ and $C$ of $A$. The proof that the induced map $\varphi_{*}: \operatorname{Har}(A, G) \longrightarrow \operatorname{Har}(A, H)$ of every homomorphism between abelian groups $\varphi: G \longrightarrow H$ is a homomorphism only uses naturality and is analogous to the proof of [16, 3.2].

Remark 2.3.6. Let $A$ be a commutative $\mathbb{S}$-algebra.

(a) The Harrison functor $\operatorname{Har}(A,-)$ restricted to abelian groups is additive: for abelian groups $G_{1}$ and $G_{2}$,

$$
\operatorname{Har}\left(A, G_{1} \times G_{2}\right) \cong \operatorname{Har}\left(A, G_{1}\right) \times \operatorname{Har}\left(A, G_{2}\right) .
$$

(b) If $G$ is an abelian group of exponent $n$, then multiplication by $n$ is induced by multiplication by $n$ on $G$ which factors through the trivial group, so

$$
n \operatorname{Har}(A, G)=0 \text {. }
$$

It would be interesting to have a better understanding of the function out of $\operatorname{Har}(A, G)$ into a subcategory of the category of $A_{*}$-algebras which sends a $G$ Galois extension $B / A$ to $B_{*} / A_{*}$. In Section 2.4 we investigate the corresponding question for $\operatorname{Har}^{w}(A, G)$ in the case where $G$ is abelian. 


\subsection{TOPOlOGicAl KUMmer TheORY}

We will now describe analogous constructions to those of Section 1.2 when the following conditions are satisfied.

Condition 2.4.1. $A$ is a commutative $\mathbb{S}$-algebra, and $G$ is a finite abelian group for which

- $A_{0}$ contains $1 /|G|$;

- $A_{0}$ contains a primitive $d$-th root of unity $\zeta$, where $d$ is the exponent of $G$;

- $A_{0}$ is connected (i.e., it has no non-trivial idempotents).

By generalizing constructions of 27] as in Example 2.2.8 we can always arrange for the second condition to hold whenever the first does. The third condition is not strictly necessary but simplifies the ensuing discussion.

Theorem 2.4.2. Suppose that $A$ and $G$ satisfy Condition 2.4.1 and let $B / A$ be a $G$-Galois extension. If for every invertible $A$-module $U, U_{*}$ is an invertible graded $A_{*}$-module, then $B_{*} / A_{*}$ is a G-Galois extension.

We will see later that the invertibility condition is not void in general. For a more thorough treatment of the question of when invertible module spectra give rise to invertible coefficients, see 5 .

Proof. Note that there is a decomposition of the form (1.2.2),

$$
A_{0}[G]=\bigoplus_{\chi} A_{0}(\chi),
$$

defined using idempotents $e_{\chi}$ as defined in (1.2.3). By Theorem1.4.10)(c), $B \wedge G_{+} \sim$ $F_{A}(B, B)$. Each $e_{\chi}$ is an element of $A_{0}[G]$ and can be realized by a map of $A$ modules $A \rightarrow A \wedge G_{+}$. Composing this with the unit of $B$ gives rise to a map

$$
A \longrightarrow B \wedge G_{+} \sim F_{A}(B, B)
$$

whose action on $B$ can be iterated to produce an $A$-module $B(\chi)=e_{\chi} B$ which is well defined up to homotopy equivalence. There is a homotopy decomposition of A-modules

$$
B \sim \bigvee_{\chi} B(\chi)
$$

As in the algebraic case, there are also pairings

$$
B\left(\chi_{1}\right) \wedge_{A} B\left(\chi_{2}\right) \longrightarrow B\left(\chi_{1} \chi_{2}\right)
$$

Now smash with $B$ and recall that $B$ is a faithful $A$-module. We can consider the extension $B \wedge_{A} B / B$ which is equivalent to $\left(\prod_{\gamma \in G} B\right) / B$. The decomposition analogous to that of (2.4.1),

$$
B \wedge_{A} B \sim \bigvee_{\chi}\left(B \wedge_{A} B\right)(\chi),
$$

is induced from that of $B$ by smashing with $B$ and

$$
\left(B \wedge_{A} B\right)(\chi) \sim B \wedge_{A}(B(\chi)) .
$$


For the product maps we also have homotopy commutative diagrams

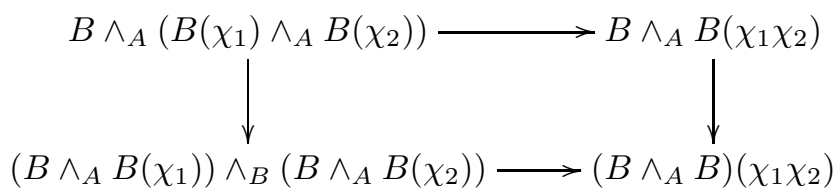

so if we can show that the bottom maps are equivalences of $B$-modules, then since $B$ is a faithful $A$-module, the maps of (2.4.2) are equivalences of $A$-modules. But the necessary verification is formally similar to that for the algebraic case proved in [16] since in homotopy there is an isomorphism of $A_{*}[G]$-modules

$$
\pi_{*}\left(B \wedge_{A} B\right) \cong \prod_{\gamma \in G} B_{*} .
$$

Thus we have shown that the map of (2.4.2) is a weak equivalence. In particular, each $B(\chi)$ is an invertible $A$-module. Now by assumption $B(\chi)_{*}$ is an invertible graded $A_{*}$-module and so is projective. From this we conclude that $B_{*}$ is a direct sum of projective $A_{*}$-modules, and so the Künneth spectral sequence collapses to give

and therefore

$$
B_{*}^{A} B \cong B_{*} \otimes_{A_{*}} B_{*},
$$

$$
B_{*} \otimes_{A_{*}} B_{*}=\prod_{\gamma \in G} B_{*} .
$$

As the order of the group is inverted in $A_{0}$ we also have

$$
\left(B_{*}\right)^{G}=\left(B^{\mathrm{h} G}\right)_{*}=A_{*},
$$

and therefore $B_{*} / A_{*}$ is a $G$-Galois extension.

Here is a reinterpretation of what we have established by combining Theorems 2.2 .4 and 2.4.2.

Theorem 2.4.3. Suppose that $A$ and $G$ satisfy Condition 2.4 .1 and that the coefficients of invertible A-modules are invertible graded $A_{*}$-modules. Then there is a natural bijection of sets

$$
\operatorname{Real}_{A, G}: \operatorname{Har}\left(A_{*}, G\right) \stackrel{\cong}{\rightrightarrows} \operatorname{Har}^{w}(A, G) .
$$

Therefore the weak Harrison set is actually a group, because it inherits the group structure from the algebraic Harrison group.

Remark 2.4.4. Note that under the above assumptions taking homotopy groups always gives a map from either Harrison set to $\operatorname{Har}\left(A_{*}, G\right)$, but for $\operatorname{Har}(A, G)$ we do not obtain an isomorphism, because we do not have an inverse map.

We close this section with an example where one can classify all of the topological Kummer extensions of an $\mathbb{S}$-algebra.

Example 2.4.5. We have

$$
\operatorname{Har}^{w}\left(K O[1 / 2], C_{2}\right) \cong C_{2} \times C_{2} \times C_{2} .
$$

To see this, first from (1.4.2) we see that

$$
K O[1 / 2]_{*}=\mathbb{Z}[1 / 2]\left[y, y^{-1}\right],
$$


where $y \in K O[1 / 2]_{4}$. As we established in [5] that invertible $K O[1 / 2]$-modules have invertible coefficients, Theorem 2.4 .3 yields

$$
\operatorname{Har}^{w}\left(K O[1 / 2], C_{2}\right) \cong \operatorname{Har}\left(\mathbb{Z}[1 / 2]\left[y, y^{-1}\right], C_{2}\right) .
$$

By Proposition 1.2.1, we find that

$$
\begin{aligned}
\operatorname{Har}^{w}\left(K O[1 / 2], C_{2}\right) & \cong\left(\mathbb{Z}[1 / 2]\left[y, y^{-1}\right]\right)^{\times} /\left(\left(\mathbb{Z}[1 / 2]\left[y, y^{-1}\right]\right)^{\times}\right)^{2} \\
& \cong C_{2} \times C_{2} \times C_{2},
\end{aligned}
$$

with generators the cosets of $-1,2, y$ with respect to $\left(\left(\mathbb{Z}[1 / 2]\left[y, y^{-1}\right]\right)^{\times}\right)^{2}$.

This leads to three non-trivial $C_{2}$-extensions of $K O[1 / 2]$ with coefficient rings

$$
K O_{*}[1 / 2, i], K O_{*}[1 / 2, \sqrt{2}], K O_{*}[1 / 2, i \sqrt{2}]
$$

which correspond to the cosets of $-1,2,-2$ and the arithmetic extensions

$$
\mathbb{Z}[1 / 2, i] / \mathbb{Z}[1 / 2], \mathbb{Z}[1 / 2, \sqrt{2}] / \mathbb{Z}[1 / 2], \mathbb{Z}[1 / 2, i \sqrt{2}] / \mathbb{Z}[1 / 2] .
$$

The extension $K U[1 / 2] / K O[1 / 2]$ corresponds to $K O_{*}[1 / 2, \sqrt{y / 2}]$. There are three more exotic extensions associated with the rings $K O_{*}[1 / 2, \sqrt{y}], K O_{*}[1 / 2, i \sqrt{2 y}]$ and $K O_{*}[1 / 2, i \sqrt{y}]$. These are the Harrison products of $K U[1 / 2]$ with the three above.

Note that we can adjoin an 8-th root of unity to $K O[1 / 2]$ and get a $C_{2} \times C_{2^{-}}$ extension (see Example 2.2.8). By taking homotopy fixed points with respect to subgroups we obtain the arithmetic $C_{2}$-extensions listed above.

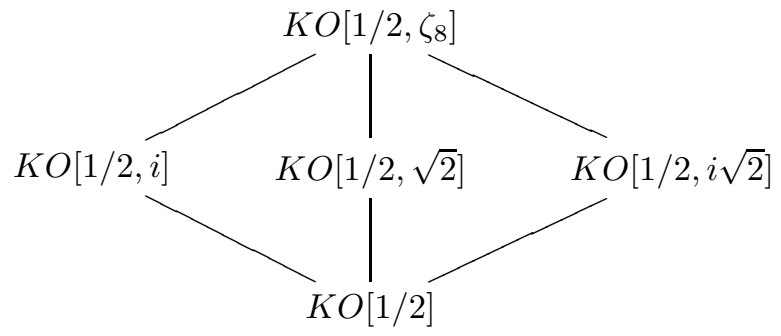

\subsection{TOPOLOGICAL ABELIAN EXTENSIONS}

In the following we will consider finite abelian extensions $B / A$ without assuming that the order of the Galois group $G$ is invertible in $A_{*}$.

Theorem 2.5.1. For every finite abelian $G$-Galois extension $B / A, B$ is an invertible $A[G]$-module.

Proof. For $G$ abelian we have a natural evaluation map

$$
\varepsilon: F_{A[G]}(B, A[G]) \wedge_{A[G]} B \longrightarrow A[G] .
$$

We will prove that this map is an equivalence. As $B$ is a faithful $A$-module, it suffices to consider the map $B \wedge_{A} \varepsilon$ instead. As $B$ is dualizable over $A$ and selfdual, we can identify $B \wedge_{A} F_{A[G]}(B, A[G]) \wedge_{A[G]} B$ with $F_{A[G]}(B, B[G]) \wedge_{A[G]} B$. Inducing up to $B$ then yields an equivalence with $F_{B[G]}\left(B \wedge_{A} B, B[G]\right) \wedge_{B[G]} B \wedge_{A} B$. 
Then $B \wedge_{A} \varepsilon$ factors as in the following diagram:

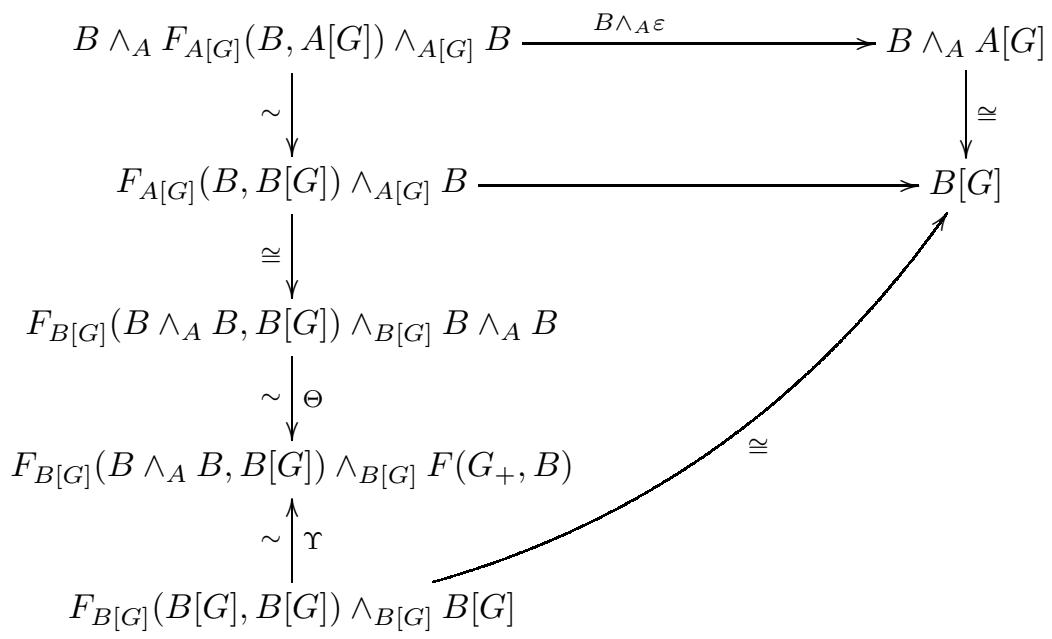

Here we use the equivalence

$$
B \wedge_{A} B \underset{\Theta}{\stackrel{\sim}{\longrightarrow}} F\left(G_{+}, B\right) \stackrel{\sim}{\longleftarrow} B[G]
$$

where $\Upsilon$ is the topological analogue of (1.1.3), in particular it is an equivalence of $B[G]$-modules.

Example 2.5.2. The result of Theorem 2.5.1 gives rise to examples of invertible $A[G]$-modules whose coefficient groups do not yield elements in the algebraic Picard group $\operatorname{Pic}\left(A_{*}[G]\right)$. Consider for instance the $C_{2}$-Galois extension $K U / K O$. We know that $K U$ is an invertible $K O\left[C_{2}\right]$-module, but $K U_{*}$ is definitely not an invertible $\mathrm{KO}_{*}\left[\mathrm{C}_{2}\right]$-module, because it is not even projective.

The Harrison group $\operatorname{Har}(A, G)$ is related to the Picard group of the group ring $A[G]$. We will make use of the constructions introduced in Proposition 2.3.5.

Theorem 2.5.3. There is a homomorphism

$$
\Psi_{G}: \operatorname{Har}(A, G) \longrightarrow \operatorname{Pic}(A[G]) .
$$

In particular, for every finite abelian group $G$ of exponent $n$, the image of $\Psi_{G}$ is contained in the $n$-torsion subgroup of $\operatorname{Pic}(A[G])$.

Proof. For a finite abelian group $G$ we define

$$
\Psi_{G}: \operatorname{Har}(A, G) \longrightarrow \operatorname{Pic}(A[G]) ; \quad \Psi_{G}([B])=[B],
$$

where the first equivalence class is in the Harrison group of $G$-Galois extensions of $A$ and the second denotes an isomorphism class in the homotopy category of $A[G]$-modules. Whenever we have to choose a representing element $B^{\prime}$ for $[B]$ in $\operatorname{Pic}(A[G])$ it will be a cofibrant $A[G]$-module. Equivalent $G$-Galois extensions over $A$ are in particular equivalent $A$-modules with $G$-action; therefore $\Psi_{G}$ is well defined.

We have to show that it is a homomorphism, i.e., that the isomorphism class of $\mu_{*}\left(B^{\prime} \wedge_{A} C^{\prime}\right)=\left(B^{\prime} \wedge_{A} C^{\prime}\right)^{\mathrm{h} K}$ coincides with that of $B^{\prime} \wedge_{A[G]} C^{\prime}$. Here $\mu: G \times G \longrightarrow G$ is the multiplication in $G$ and $K=\operatorname{ker} \mu$, while $B^{\prime}$ and $C^{\prime}$ are $A[G]$-cofibrant models of $B$ and $C$, respectively. 
If $M$ is a cofibrant $A$-module, then $E G_{+} \wedge M$ is a cofibrant model for $M$ in the category of $A[G]$-modules: the fibrations and weak equivalences in the categories $\mathscr{M}_{A}$ and $\mathscr{M}_{A[G]}$ are defined via the forgetful functor to $\mathscr{M}_{\mathbb{S}}$. Therefore if $U$ denotes the forgetful functor from $\mathscr{M}_{A[G]}$ to $\mathscr{M}_{A}$, the lifting diagram

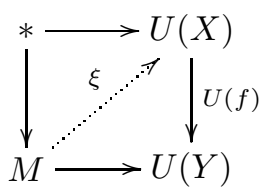

for any acyclic fibration $f: X \longrightarrow Y$ in $\mathscr{M}_{A[G]}$ has a $G$-equivariant extension

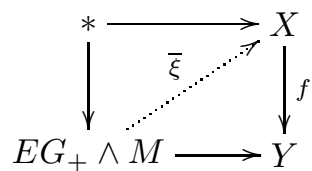

and therefore we can identify $B^{\prime} \wedge_{A[G]} C^{\prime}$ with $E G_{+} \wedge B \wedge_{A[G]} E G_{+} \wedge C$ and this in turn is equivalent to $\left(E(G \times G)_{+} \wedge B \wedge_{A} C\right) / K$. We are left with the identification of the homotopy orbits $\left(E(G \times G)_{+} \wedge B \wedge_{A} C\right) / K=\left(B \wedge_{A} C\right)_{\mathrm{h} K}$ and the homotopy fixed points.

As $B$ and $C$ are both dualizable over $A$, we obtain the chain of identifications

$$
\begin{aligned}
\left(B \wedge_{A} C\right) \wedge_{A}\left(B \wedge_{A} C\right)_{\mathrm{h} K} & \sim\left(B \wedge_{A} C \wedge_{A} B \wedge_{A} C\right)_{\mathrm{h} K} \sim\left(\prod_{G \times G}\left(B \wedge_{A} C\right)\right)_{\mathrm{h} K} \\
& \sim\left(\prod_{G \times G}\left(B \wedge_{A} C\right)\right)^{\mathrm{h} K} \sim\left(B \wedge_{A} C\right) \wedge_{A}\left(B \wedge_{A} C\right)^{\mathrm{h} K},
\end{aligned}
$$

and this shows that $\left(B \wedge_{A} C\right)_{\mathrm{h} K} \sim\left(B \wedge_{A} C\right)^{\mathrm{h} K}$, since $B \wedge_{A} C$ is faithful over $A$. Here we use the fact that $\prod_{G \times G}\left(B \wedge_{A} C\right)$ is equivalent to a wedge of copies of $F\left(K_{+}, B \wedge_{A} C\right)$ which is a free $K$-spectrum, and hence it has a trivial Tate spectrum [14, Proposition 2.4].

By Remark 2.3.6(b), when $G$ is an abelian group of exponent $n$, the image of $\Psi_{G}$ is contained in the $n$-torsion subgroup of $\operatorname{Pic}(A[G])$.

\subsection{Units of Galois extensions}

One instance of Hilbert's theorem 90 involves the vanishing of the first cohomology of Galois groups with coefficients in the units of a field extension and the straightforward identification of the fixed points of the units of the extension with the units in the base field. Note that for a general $G$-Galois extension of rings $S / R$ Hilbert's theorem 90 does not hold: instead of a vanishing result for the first group cohomology, there is an exact sequence [7, §5],

$$
\begin{aligned}
0 \rightarrow \mathrm{H}^{1}\left(G, S^{\times}\right) \longrightarrow \operatorname{Pic}(R) & \longrightarrow \mathrm{H}^{0}(G, \operatorname{Pic}(S)) \longrightarrow \mathrm{H}^{2}\left(G, S^{\times}\right) \\
& \longrightarrow \operatorname{Br}(S / R) \longrightarrow \mathrm{H}^{1}(G, \operatorname{Pic}(S)) \longrightarrow \mathrm{H}^{3}\left(G, S^{\times}\right),
\end{aligned}
$$

in which $\operatorname{Br}(S / R)$ is the relative Brauer group. Examples of Galois extensions with non-trivial $\mathrm{H}^{1}\left(G, S^{\times}\right)$are mentioned in [7, 5.5(d)]. We will prove a version of Hilbert's theorem 90 corresponding to the classical statement for invariants.

In the following we use the concept of units of ring spectra. These were introduced by Patterson, Stong and Waldhausen, and their multiplicative properties 
were developed in 20. More material on these is contained in the notes [2], and we are grateful to $\mathrm{M}$. Ando for providing them.

Definition 2.6.1. Let $R$ be a ring spectrum. The units $G L_{1}(R)$ of the ring spectrum $R$ are defined via the following homotopy pullback square:

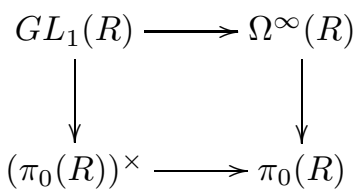

in which $\Omega^{\infty}(R)$ denotes the underlying infinite loop space of the $\Omega$-spectrum associated to $R$, i.e., the zeroth space of the spectrum $R$.

The quotient map from the space $\Omega^{\infty}(R)$ to its path components is a fibration. Therefore the units of $R$ are given by an actual pullback square.

Now assume that $R$ possesses an action of some finite group $G$ by maps of ring spectra. More precisely, let $R$ be a naive $G$-spectrum with a coherent $E_{\infty}$-structure in the sense of [19, VII, Def. 2.1]. We recall from [19, VII, Proposition 2.8] that the zeroth space $R(0)=\Omega^{\infty}(R)$ inherits a $G$ - $E_{\infty}$-structure from $R$.

The homotopy groups of $R$ inherit the $G$-action as well. As everything takes place in a setting of $\Omega$-spectra, the zeroth homotopy group $\pi_{0}(R)$ is given by $\pi_{0}\left(\Omega^{\infty}(R)\right)$. The inclusion of the units into the full ring $\pi_{0}(R)$ is clearly $G$-equivariant, and so is the quotient map from $\Omega^{\infty}(R)$ to $\pi_{0}(R)$.

Theorem 2.6.2. Assume that $R$ is a G-ring spectrum as above and for which $\pi_{0}\left(R^{\mathrm{h} G}\right) \cong\left(\pi_{0}(R)\right)^{G}$. Then the homotopy fixed points of the units $G L_{1}(R)$ are given by the units of $R^{\mathrm{h} G}$.

Proof. Taking the zeroth space of a spectrum commutes with homotopy fixed points, because using the setting of [19, I, §3] we have the following chain of identifications:

$$
\begin{aligned}
\left(\Omega^{\infty}(R)\right)^{\mathrm{h} G} & =F\left(E G_{+}, \Omega^{\infty}(R)\right)^{G}=F\left(E G_{+}, R(0)\right)^{G} \\
& =\left(F\left(E G_{+}, R\right)(0)\right)^{G}=\left(F\left(E G_{+}, R\right)^{G}\right)(0) \\
& =\Omega^{\infty}\left(R^{\mathrm{h} G}\right) .
\end{aligned}
$$

By assumption, the homotopy fixed points of the discrete set $\pi_{0}(R)$ are

$$
F\left(E G_{+}, \pi_{0}(R)\right)^{G} \cong\left(\pi_{0}(R)\right)^{G} \cong \pi_{0}\left(F\left(E G_{+}, R\right)^{G}\right),
$$

and therefore the pullback for the homotopy fixed points of $G L_{1}(R)$ is the pullback of the diagram

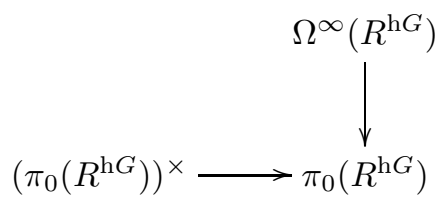

and by definition this is $G L_{1}\left(R^{\mathrm{h} G}\right)$.

We now obtain a topological version of Hilbert's theorem 90 as an immediate consequence of the above result. Note that the following result also holds if $B$ is ramified over $A$. 
Corollary 2.6.3. Let $G$ be a finite group and let $A \longrightarrow B$ be a weak $G$-Galois extension with $\left(\pi_{0}(B)\right)^{G} \cong \pi_{0}(A)$. Then

$$
G L_{1}(B)^{\mathrm{h} G} \sim G L_{1}(A) .
$$

The condition on the zeroth homotopy group is of course satisfied in the case of a realization of an algebraic $G$-Galois extension of a commutative $\mathbb{S}$-algebra. In the special case of Eilenberg-Mac Lane spectra $H R \longrightarrow H S$ the result gives the classical identity $\left(S^{\times}\right)^{G} \cong R^{\times}$. The $C_{2}$-Galois extension $K U / K O$ also satisfies the condition on $\pi_{0}$ and so do the naturally occurring examples in [26, §5]. However, there are examples where this condition is not satisfied: take $A$ to be $\bigvee_{n \in \mathbb{Z}} \Sigma^{2 n} K O$ and take $B$ to be

$$
\bigvee_{n \in \mathbb{Z}} \Sigma^{2 n} K O \wedge_{K O} K U \sim \bigvee_{n \in \mathbb{Z}} \Sigma^{2 n} K U
$$

Therefore $\pi_{0}(B)^{C_{2}}$ consists of copies of the integers, whereas $\pi_{0}(A)$ contains summands $\mathbb{Z} / 2 \mathbb{Z}$.

\section{REFERENCES}

[1] J. F. Adams, Stable Homotopy and Generalised Homology, University of Chicago Press (1974). MR0402720 (53:6534)

[2] M. Ando, The units of a ring spectrum and Thom spectra, unpublished notes (2004).

[3] M. Auslander \& O. Goldman, The Brauer group of a commutative ring, Trans. Amer. Math. Soc. 97 (1960), 367-409. MR0121392 (22:12130)

[4] A. Baker \& B. Richter, On the $\Gamma$-cohomology of rings of numerical polynomials and $E_{\infty}$ structures on $K$-theory, Comm. Math. Helv. 80 (2005), 691-723. MR2182697

[5] _ Invertible modules for commutative $\mathbb{S}$-algebras with residue fields, Manuscripta Mathematica 118 (2005), 99-119. MR2171294(2006f:55009)

[6] M. Basterra \& B. Richter, (Co)homology theories for commutative ( $S$-)algebras, in 'Structured Ring Spectra', eds. A. Baker \& B. Richter, London Math. Lecture Notes 315, Cambridge University Press (2004), 115-131. MR2122156 (2005j:55003)

[7] S. U. Chase, D. K. Harrison \& A. Rosenberg, Galois theory and Galois cohomology of commutative rings, Mem. Amer. Math. Soc. 52 (1965), 15-33. MR0195922 (33:4118)

[8] E. S. Devinatz \& M. J. Hopkins, Homotopy fixed point spectra for closed subgroups of the Morava stabilizer groups, Topology, 43 (2004), 1-47. MR2030586 (2004i:55012)

[9] A. Dold \& D. Puppe, Duality, trace, and transfer, Proceedings of the International Conference on Geometric Topology (Warsaw, 1978), PWN (1980), 81-102; also appeared in Proc. Steklov Inst. Math. 154 (1985), 85-103.

[10] W. G. Dwyer, D. M. Kan, \& J. H. Smith, Homotopy commutative diagrams and their realizations, J. Pure Appl. Algebra 57 (1989), 5-24. MR0984042 (90d:18007)

[11] A. Elmendorf, I. Kriz, M. Mandell \& J. P. May, Rings, modules, and algebras in stable homotopy theory, Mathematical Surveys and Monographs 47 (1997). MR:1417719 (97h:55006)

[12] M. Ferrero \& A. Paques, Galois theory of commutative rings revisited, Beiträge Algebra Geom. 38 (1997), 399-410. MR.1473117 (98i:13010)

[13] P. G. Goerss \& M. J. Hopkins, Moduli spaces of commutative ring spectra, in 'Structured Ring Spectra', eds. A. Baker \& B. Richter, London Math. Lecture Notes 315, Cambridge University Press (2004), 151-200. MR2125040 (2006b:55010)

[14] J. P. C. Greenlees \& J. P. May, Generalized Tate cohomology, Mem. Amer. Math. Soc. 113 (1995) no. 543. MR.1230773 (96e:55006)

[15] C. Greither, Cyclic Galois extensions and normal bases, Trans. Amer. Math. Soc. 326 (1991), 307-343. MR1014248 (91j:11093)

[16] Cyclic Galois extensions of commutative rings, Lecture Notes in Mathematics 1534 (1992). MR.1222646 (94k:11121)

[17] D. K. Harrison, Abelian extensions of commutative rings, Mem. Amer. Math. Soc. No. 52 (1965), 1-14. MR0195921 (33:4117)

[18] M. Hovey, J. H. Palmieri \& N. P. Strickland, Axiomatic stable homotopy theory, Mem. Amer. Math. Soc. 128 (1997), no. 610. MR1388895 (98a:55017) 
[19] L. G. Lewis, J. P. May, M. Steinberger \& J. E. McClure, Equivariant stable homotopy theory, Lecture Notes in Mathematics 1213 (1986). MR0866482 (88e:55002)

[20] J. P. May with contributions by F. Quinn, N. Ray and J. Tornehave, $E_{\infty}$ ring spaces and $E_{\infty}$ ring spectra, Lecture Notes in Mathematics 577 (1977). MR0494077 (58:13008)

[21] Equivariant Homotopy and Cohomology theories, CBMS Regional Conference Series in Mathematics 91 (1996).

[22] _ Picard groups, Grothendieck rings, and Burnside rings of categories, Advances in Math. 163 (2001), 1-16. MR1867201(2002k:18011)

[23] A. Robinson, Gamma homology, Lie representations and $E_{\infty}$ multiplications, Invent. Math. 152 (2003), 331-348. MR 1974890(2004c:55020)

[24] _ Classical obstructions and $S$-algebras, in 'Structured Ring Spectra', eds. A. Baker \& B. Richter, London Math. Lecture Notes 315, Cambridge University Press (2004), 133-149. MR2122157 (2005k:55007)

[25] A. Robinson \& S. Whitehouse, Operads and $\Gamma$-homology of commutative rings, Math. Proc. Camb. Phil. Soc. 132 (2002), 197-234. MR.1874215 (2002j:18011)

[26] J. Rognes, Galois extensions of structured ring spectra, preprint math.AT/0502183, to appear in the Memoirs of the Amer. Math. Soc.

[27] R. Schwänzl, R. M. Vogt \& F. Waldhausen, Adjoining roots of unity to $E_{\infty}$ ring spectra in good cases - a remark, Contemp. Math. 239, AMS (1999), 245-249. MR1718085 (2001b:55026)

[28] C. A. Weibel, An Introduction to Homological Algebra, Cambridge University Press (1994). MR:1269324 (95f:18001)

Department of Mathematics, University of Glasgow, Glasgow G12 8QW, Scotland

E-mail address: a.baker@maths.gla.ac.uk

URL: http://www.maths.gla.ac.uk/ ajb

Fachbereich Mathematik der Universität Hamburg, Bundesstrasse 55, 20146 HamBURG, Germany

E-mail address: richter@math.uni-hamburg.de

URL: http://www.math.uni-hamburg.de/home/richter/ 